Article

\title{
Nonlocal Elasticity Response of Doubly-Curved Nanoshells
}

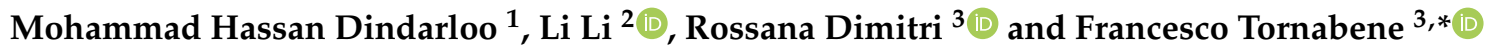 \\ 1 Department of Mechanical Engineering, Tarbiat Modares University, Tehran 14115-111, Iran; \\ mhdmhd74@yahoo.com \\ 2 State Key Lab of Digital Manufacturing Equipment and Technology, School of Mechanical Science and \\ Engineering, Huazhong University of Science and Technology, Wuhan 430074, China; lili_em@hust.edu.cn \\ 3 Department of Innovation Engineering, University of Salento, 73100 Lecce, Italy; \\ rossana.dimitri@unisalento.it \\ * Correspondence: francesco.tornabene@unisalento.it
}

Received: 2 February 2020; Accepted: 10 March 2020; Published: 16 March 2020

check for updates

\begin{abstract}
In this paper, we focus on the bending behavior of isotropic doubly-curved nanoshells based on a high-order shear deformation theory, whose shape functions are selected as an accurate combination of exponential and trigonometric functions instead of the classical polynomial functions. The small-scale effect of the nanostructure is modeled according to the differential law consequent, but is not equivalent to the strain-driven nonlocal integral theory of elasticity equipped with Helmholtz's averaging kernel. The governing equations of the problem are obtained from the Hamilton's principle, whereas the Navier's series are proposed for a closed form solution of the structural problem involving simply-supported nanostructures. The work provides a unified framework for the bending study of both thin and thick symmetric doubly-curved shallow and deep nanoshells, while investigating spherical and cylindrical panels subjected to a point or a sinusoidal loading condition. The effect of several parameters, such as the nonlocal parameter, as well as the mechanical and geometrical properties, is investigated on the bending deflection of isotropic doubly-curved shallow and deep nanoshells. The numerical results from our investigation could be considered as valid benchmarks in the literature for possible further analyses of doubly-curved applications in nanotechnology.
\end{abstract}

Keywords: doubly-curved nanoshells; high-order shear deformation theory; nonlocal elasticity theory; static analysis

\section{Introduction}

Doubly-curved shells are three-dimensional structures, commonly used in many engineering applications, such as aerospace structures, airplane vehicles, or big constructions such as stadium cupolas. In their service life, doubly-curved shells are usually affected by different kinds of loading conditions due to their special geometrical shapes. Therefore, the knowledge of their static response is a crucial subject of investigation, especially from an applied design standpoint. The application of adequate numerical models for the bending analysis of doubly-curved shell structures represents a key aspect to be investigated, which is done herein for nanoshell applications. Doubly-curved shells can feature a complex geometry, which makes the exact description of their mathematical problem difficult. Based on the available literature, several theories have been developed to handle the mechanical behavior of complex shell structures, namely, the 3D elasticity [1-3], the Equivalent Single Layer (ESL) theories [4-7], and the Layer Wise (LW) [8-11] theories. In what follows, we propose an ESL model for the static analysis of composite nanoshells with high mechanical magnetic, electronic, and biomedical properties. In this context, classical continuum theories are known to be unable to accurately 
analyze microstructures or nanostructures and their size effect, whereas the nonlocal theories are more appropriate to account for small scale effects [12-14], together with the physical interactions of atoms and molecules at a microscale or nanoscale.

In recent years, a large number of works has focused on the structural behavior of beams, plates, and shell structures, as briefly reviewed in the following. Ramirez et al. [15] investigated the static analysis of functionally graded (FG) elastic anisotropic plates using a discrete layer approach together with the Ritz method. Merdaci and Belghoul [16] applied a higher-order shear deformation theory (HSDT) with trigonometric shear strain shape functions to study the statics of FG plates with porosities, while assuming the Navier's series to solve the equations of motion. In line with the previous works, Alibeigloo and Nouri [17] studied the statics of FG cylindrical shell with piezoelectric layers by using the differential quadrature method to handle the governing differential equations and boundary conditions. A standard finite element approach was differently applied by Kumar et al. [18] for the static and dynamic analysis of composite cylindrical shells based on the first-order shear deformation theory (FSDT). A nonlinear analysis of the structural response of FG shells was also performed by Frikha and Dammak [19] through the application of discrete double director shell elements. Thin and thick shell theories were proposed alternatively, according to a HSDT, where the material properties were graded throughout the thickness, according to a simple power-law. Moreover, Mantari et al. [20] proposed a novel HSDT to investigate the static and dynamic response of laminated composite and sandwich plates and shells with different geometries. They considered the transverse shear strain field throughout the thickness, along with the tangential stress-free boundary conditions on the shell surface. The governing equations of the problem and the associated boundary conditions were derived by the principle of virtual work whose solution was determined numerically by means of the Navier series.

As far as nanotechnology is concerned, an increased number of works in literature has focused on the nonlocal mechanics of nanostructures [21-26]. More specifically, Sahmani and Aghdam [21] investigated the nonlinear instability of hydrostatic pressurized hybrid nanoshells based on nonlocal elasticity theories, combined to the HSDT. Zeighampour et al. [22] employed a strain gradient theory to investigate the torsional vibrations and static behavior of cylindrical shells, whose equations of motion and non-classical boundary conditions were derived, according to the Hamilton's principle. A further application of the nonlocal elasticity theory can be found in Reference [23] for a parametric study of the axial post-buckling behavior of nanoshells with different nonlocal parameters. Various nonlocal theories have been applied within coupled problems, such as piezoelectric, flexoelectric, or thermo-electro-mechanical shells at different scales both for simple [24-32] or more complex [33-46] geometries.

Based on the available literature, however, limited attention has been paid to the nonlocal mechanical behavior of symmetric doubly-curved deep nanoshells. This is explored for isotropic doubly-curved nanoshells, where we propose a novel nonlocal shear deformation theory, based on a combination of exponential and trigonometric functions. These functions are selected for their higher accuracy compared to the polynomial functions [47]. The small-scale effect of the nanostructure is, thus, modeled, according to a differential law consequent, but not equivalent to the strain-driven nonlocal integral theory of elasticity supplemented with Helmholtz's averaging kernel, whereby the strain-displacement relations for symmetric nanoshells are based on the Reddy's doubly-curved shells theory. The governing equations and boundary conditions are derived by the Hamilton's principle whose theoretical formulation is detailed in Section 2. In Section 3, we propose a Navier-type procedure to solve the problem in a closed-form, whose accuracy is checked against the open literature in Section 4, along with a systematic investigation aimed at studying the influence of nonlocal and geometrical parameters on the deflection response of both shallow and deep doubly-curved isotropic nanoshells. The main conclusive remarks are discussed in the last section, which could be of great interest for scientists and designers for many practical applications. 


\section{Governing Equations of Doubly-Curved Nanoshells}

In this section, we provide a brief overview of the mathematical fundamentals and governing equations of the problem with a special focus on the nonlocal structural response of shallow and deep isotropic doubly-curved nanoshells. The displacement field is modeled based on the combination of exponential, sinusoidal, and cosine strain functions due to their accuracy, as verified in Reference [47]. Figure 1 shows the geometrical scheme of the doubly-curved nanoshell analyzed in this scenario, where $h$ is the thickness of the nanoshell, $R_{1}$ and $R_{2}$ refer to its curvature radii, $a$ and $b$ stand for its width and length, respectively.

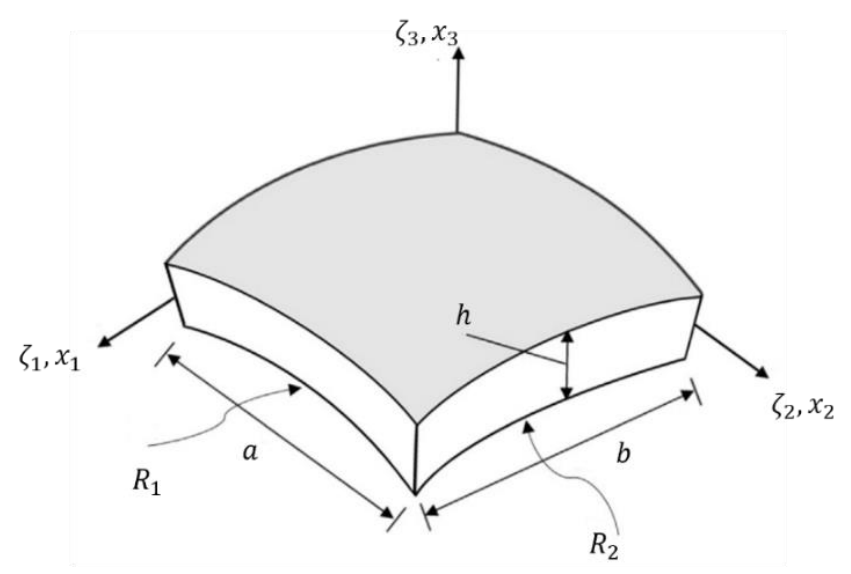

Figure 1. Geometrical scheme of a doubly-curved nanoshell.

Based on the HSDT [20], the displacement field of doubly-curved deep nanoshells are expressed by the equation below.

$$
\begin{aligned}
& \bar{u}\left(\xi_{1}, \xi_{2}, \xi_{3}, t\right)=\left(1+\frac{\xi_{3}}{R_{1}}\right) u\left(\xi_{1}, \xi_{2}, t\right)+\xi_{3}\left(y^{*} \phi_{1}-\frac{\partial w\left(\xi_{1}, \xi_{2}, t\right)}{a_{1} \partial \xi_{1}}\right)+f\left(\xi_{3}\right) \phi_{1}\left(\xi_{1}, \xi_{2}, t\right) \\
& \bar{v}\left(\xi_{1}, \xi_{2}, \xi_{3}, t\right)=\left(1+\frac{\xi_{3}}{R_{2}}\right) v\left(\xi_{1}, \xi_{2}, t\right)+\xi_{3}\left(y^{*} \phi_{2}-\frac{\partial w\left(\xi_{1}, \xi_{2}, t\right)}{a_{2} \partial \xi_{2}}\right)+f\left(\xi_{3}\right) \phi_{2}\left(\xi_{1}, \xi_{2}, t\right) \\
& \bar{w}\left(\xi_{1}, \xi_{2}, \xi_{3}, t\right)=w\left(\xi_{1}, \xi_{2}, t\right)
\end{aligned}
$$

where $\bar{u}\left(\xi_{1}, \xi_{2}, \xi_{3}, t\right), \bar{v}\left(\xi_{1}, \xi_{2}, \xi_{3}, t\right)$ and $\bar{w}\left(\xi_{1}, \xi_{2}, \xi_{3}, t\right)$ are the displacement components along the $\xi_{1}$, $\xi_{2}$ and $\xi_{3}$ directions, respectively. $u\left(\xi_{1}, \xi_{2}, t\right), v\left(\xi_{1}, \xi_{2}, t\right)$ and $w\left(\xi_{1}, \xi_{2}, t\right)$ refer to the displacement field at the mid-surface. $y^{*}=m \pi / h$ is defined as in Reference [48], whereby the value $m=0.5$ produces the closest response to a 3D elasticity bending solution. Moreover, $\phi_{1}$ and $\phi_{2}$ denote the rotations about $\xi_{1}$ and $\xi_{2}$ axes, respectively. For an accurate study of the bending response of the doubly-curved deep nanoshells, a combination of the exponential and trigonometric shape functions is proposed within the formulation.

$$
f\left(\xi_{3}\right)=\frac{\pi h}{\pi^{4}+h^{4}} e^{\left(\frac{h \xi_{3}}{\pi}\right)}\left(\pi^{2} \sin \left(\frac{h \xi_{3}}{\pi}\right)+h^{2} \cos \left(\frac{h \xi_{3}}{\pi}\right)\right)-\frac{\pi h^{3}}{\pi^{4}+h^{4}}
$$

This is in view of the lower accuracy of polynomial functions [47]. The selected shape function follows the shear deformation distribution throughout the thickness, and satisfies the shear stress-free surface conditions without considering any shear correction factor. Note that, due to the introduction of the shear deformation effect, both thin and thick shell structures are treated in a unified framework.

For a doubly-curved deep shell, the strain-displacement relations can be expressed by the formula below [49]. 


$$
\begin{aligned}
\varepsilon_{1} & =\frac{1}{A_{1}}\left(\frac{\partial \bar{u}}{\partial \xi_{1}}+\frac{1}{a_{2}} \frac{\partial a_{1}}{\partial \xi_{2}} \bar{v}+\frac{a_{1}}{R_{1}} \bar{w}\right) \\
\varepsilon_{2} & =\frac{1}{A_{2}}\left(\frac{\partial \bar{v}}{\partial \xi_{2}}+\frac{1}{a_{1}} \frac{\partial a_{2}}{\partial \xi_{1}} \bar{u}+\frac{a_{2}}{R_{2}} \bar{w}\right) \\
\varepsilon_{6} & =\frac{A_{2}}{A_{1}} \frac{\partial}{\partial \xi_{1}}\left(\frac{\bar{v}}{A_{2}}\right)+\frac{A_{1}}{A_{2}} \frac{\partial}{\partial \xi_{2}}\left(\frac{\bar{u}}{A_{1}}\right) \\
\varepsilon_{4} & =\frac{1}{A_{2}} \frac{\partial \bar{w}}{\partial \xi_{2}}+A_{2} \frac{\partial}{\partial \xi_{3}}\left(\frac{\bar{v}}{A_{2}}\right) \\
\varepsilon_{5} & =\frac{1}{A_{1}} \frac{\partial \bar{w}}{\partial \xi_{1}}+A_{1} \frac{\partial}{\partial \xi_{3}}\left(\frac{\bar{u}}{A_{1}}\right)
\end{aligned}
$$

where

$$
A_{1}=\left(1+\frac{\xi_{3}}{R_{1}}\right) a_{1} ; A_{2}=\left(1+\frac{\xi_{3}}{R_{2}}\right) a_{2}
$$

$a_{1}$ and $a_{2}$ representing the tangent vectors along the $\xi_{1}$ and $\xi_{2}$ directions, respectively, and $\varepsilon_{i}=(i=1,2, \ldots, 6)$ are strain components.

The doubly-curved shallow nanoshell represents a limit case when it is possible to neglect $\xi_{3}$ due to its small dimension compared to the curvature radii, i.e.,

$$
\begin{aligned}
& \frac{\xi_{3}}{R_{1}} \rightarrow 0 \Rightarrow 1+\frac{\xi_{3}}{R_{1}} \rightarrow 1 \Rightarrow A_{1}=a_{1} \\
& \frac{\xi_{3}}{R_{2}} \rightarrow 0 \Rightarrow 1+\frac{\xi_{3}}{R_{2}} \rightarrow 1 \Rightarrow A_{2}=a_{2}
\end{aligned}
$$

By substituting Equation (1) into Equation (3), we get the following relations.

$$
\begin{aligned}
& \varepsilon_{1}=\frac{1}{A_{1}}\left(\begin{array}{l}
\left(1+\frac{\xi_{3}}{R_{1}}\right) \frac{\partial u}{\partial \xi_{1}}+\xi_{3}\left(y^{*} \frac{\partial \phi_{1}}{\partial \xi_{1}}-\frac{\partial^{2} w}{a_{1} \partial \xi_{1}^{2}}\right)+f\left(\xi_{3}\right) \frac{\partial \phi_{1}}{\partial \xi_{1}}+ \\
+\frac{1}{a_{2}} \frac{\partial a_{1}}{\partial \xi_{2}}\left(\left(1+\frac{\xi_{3}}{R_{2}}\right) v+\xi_{3}\left(y^{*} \phi_{2}-\frac{\partial w}{a_{2} \partial \xi_{2}}\right)+f\left(\xi_{3}\right) \phi_{2}\right)+\frac{a_{1}}{R_{1}} w
\end{array}\right) \\
& \varepsilon_{2}=\frac{1}{A_{2}}\left(\begin{array}{l}
\left(1+\frac{\xi_{3}}{R_{2}}\right) \frac{\partial v}{\partial \xi_{2}}+\xi_{3}\left(y^{*} \frac{\partial \phi_{2}}{\partial \xi_{2}}-\frac{\partial^{2} w}{a_{2} \partial \xi_{2}^{2}}\right)+f\left(\xi_{3}\right) \frac{\partial \phi_{2}}{\partial \xi_{2}}+ \\
+\frac{1}{a_{1}} \frac{\partial a_{2}}{\partial \xi_{1}}\left(\left(1+\frac{\xi_{3}}{R_{1}}\right) u+\xi_{3}\left(y^{*} \phi_{1}-\frac{\partial w}{a_{1} \partial \xi_{1}}\right)+f\left(\xi_{3}\right) \phi_{1}\right)+\frac{a_{2}}{R_{2}} w
\end{array}\right) \\
& \varepsilon_{6}=\frac{1}{A_{1}}\left(\left(1+\frac{\xi_{3}}{R_{2}}\right) \frac{\partial v}{\partial \xi_{1}}+\xi_{3}\left(y^{*} \frac{\partial \phi_{2}}{\partial \xi_{1}}-\frac{\partial^{2} w}{a_{2} \partial \xi_{1} \partial \xi_{2}}\right)+f\left(\xi_{3}\right) \frac{\partial \phi_{2}}{\partial \xi_{1}}\right)+ \\
& +\frac{1}{A_{2}}\left(\left(1+\frac{\xi_{3}}{R_{1}}\right) \frac{\partial u}{\partial \xi_{2}}+\xi_{3}\left(y^{*} \frac{\partial \phi_{1}}{\partial \xi_{2}}-\frac{\partial^{2} w}{a_{1} \partial \xi_{1} \partial \xi_{2}}\right)+f\left(\xi_{3}\right) \frac{\partial \phi_{1}}{\partial \xi_{2}}\right) \\
& \varepsilon_{4}=\frac{1}{A_{2}} \frac{\partial w}{\partial \xi_{2}}+\frac{1}{R_{2}} v+y^{*} \phi_{2}-\frac{\partial w}{a_{2} \partial \xi_{2}}+\frac{d f\left(\xi_{3}\right)}{d \xi_{3}} \phi_{2} \\
& \varepsilon_{5}=\frac{1}{A_{1}} \frac{\partial w}{\partial \xi_{1}}+\frac{1}{R_{1}} u+y^{*} \phi_{1}-\frac{\partial w}{a_{1} \partial \xi_{1}}+\frac{d f\left(\xi_{3}\right)}{d \xi_{3}} \phi_{1}
\end{aligned}
$$

The size effect of doubly-curved nanoshells starts considering the strain-driven gradient model by Eringen [13] to include possible nonlocal long-range interactions, which is also discussed for beam applications in References [50-53]. Thus, the stress-strain relations for both thin and thick isotropic nanoshells, accounting for small effects, are expressed by the equation below

$$
\left(1-\mu^{2} \nabla^{2}\right)\left\{\begin{array}{c}
\sigma_{1} \\
\sigma_{2} \\
\sigma_{6} \\
\sigma_{4} \\
\sigma_{5}
\end{array}\right\}=\left[\begin{array}{ccccc}
Q_{11} & Q_{12} & 0 & 0 & 0 \\
Q_{12} & Q_{22} & 0 & 0 & 0 \\
0 & 0 & Q_{66} & 0 & 0 \\
0 & 0 & 0 & Q_{44} & 0 \\
0 & 0 & 0 & 0 & Q_{55}
\end{array}\right]\left\{\begin{array}{c}
\varepsilon_{1} \\
\varepsilon_{2} \\
\varepsilon_{6} \\
\varepsilon_{4} \\
\varepsilon_{5}
\end{array}\right\}
$$


where

$$
Q_{11}=Q_{22}=\frac{E}{1-v^{2}} \quad, \quad Q_{12}=\frac{v E}{1-v^{2}} \quad, \quad Q_{44}=Q_{55}=Q_{66}=G
$$

$\mu$ is the nonlocal parameter, $E$ and $G$ stand for the Young's modulus and shear modulus of the nanoshell, respectively, and $v$ is the Poisson's ratio. By combining Equations (6) and (7), we get the following.

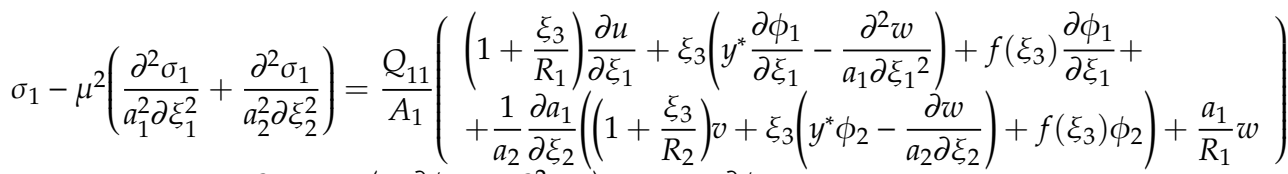

$$
\begin{aligned}
& +\frac{Q_{12}}{A_{2}}\left(\begin{array}{l}
\left(1+\frac{\xi_{3}}{R_{2}}\right) \frac{\partial v}{\partial \xi_{2}}+\xi_{3}\left(y^{*} \frac{\partial \phi_{2}}{\partial \xi_{2}}-\frac{\partial^{2} w}{a_{2} \partial \xi_{2}^{2}}\right)+f\left(\xi_{3}\right) \frac{\partial \phi_{2}}{\partial \xi_{2}}+ \\
+\frac{1}{a_{1}} \frac{\partial a_{2}}{\partial \xi_{1}}\left(\left(1+\frac{\xi_{3}}{R_{1}}\right) u+\xi_{3}\left(y^{*} \phi_{1}-\frac{\partial w}{a_{1} \partial \xi_{1}}\right)+f\left(\xi_{3}\right) \phi_{1}\right)+\frac{a_{2}}{R_{2}} w
\end{array}\right) \\
& \sigma_{2}-\mu^{2}\left(\frac{\partial^{2} \sigma_{2}}{a_{1}^{2} \partial \xi_{1}^{2}}+\frac{\partial^{2} \sigma_{2}}{a_{2}^{2} \partial \xi_{2}^{2}}\right)=\frac{Q_{12}}{A_{1}}\left(\begin{array}{l}
\left(1+\frac{\xi_{3}}{R_{1}}\right) \frac{\partial u}{\partial \xi_{1}}+\xi_{3}\left(y^{*} \frac{\partial \phi_{1}}{\partial \xi_{1}}-\frac{\partial^{2} w}{a_{1} \partial \xi_{1}^{2}}\right)+f\left(\xi_{3}\right) \frac{\partial \phi_{1}}{\partial \xi_{1}}+ \\
+\frac{1}{a_{2}} \frac{\partial a_{1}}{\partial \xi_{2}}\left(\left(1+\frac{\xi_{3}}{R_{2}}\right) v+\xi_{3}\left(y^{*} \phi_{2}-\frac{\partial w}{a_{2} \partial \xi_{2}}\right)+f\left(\xi_{3}\right) \phi_{2}\right)+\frac{a_{1}}{R_{1}} w
\end{array}\right) \\
& +\frac{Q_{22}}{A_{2}}\left(\begin{array}{l}
\left(1+\frac{\xi_{3}}{R_{2}}\right) \frac{\partial v}{\partial \xi_{2}}+\xi_{3}\left(y^{*} \frac{\partial \phi_{2}}{\partial \xi_{2}}-\frac{\partial^{2} w}{a_{2} \partial \xi_{2}^{2}}\right)+f\left(\xi_{3}\right) \frac{\partial \phi_{2}}{\partial \xi_{2}}+ \\
+\frac{1}{a_{1}} \frac{\partial a_{2}}{\partial \xi_{1}}\left(\left(1+\frac{\xi_{3}}{R_{1}}\right) u+\xi_{3}\left(y^{*} \phi_{1}-\frac{\partial w}{a_{1} \partial \xi_{1}}\right)+f\left(\xi_{3}\right) \phi_{1}\right)+\frac{a_{2}}{R_{2}} w
\end{array}\right) \\
& \sigma_{6}-\mu^{2}\left(\frac{\partial^{2} \sigma_{6}}{a_{1}^{2} \partial \xi_{1}^{2}}+\frac{\partial^{2} \sigma_{6}}{a_{2}^{2} \partial \xi_{2}^{2}}\right)=\frac{Q_{66}}{A_{1}}\left(\left(1+\frac{\xi_{3}}{R_{2}}\right) \frac{\partial v}{\partial \xi_{1}}+\xi_{3}\left(y^{*} \frac{\partial \phi_{2}}{\partial \xi_{1}}-\frac{\partial^{2} w}{a_{2} \partial \xi_{1} \partial \xi_{2}}\right)+f\left(\xi_{3}\right) \frac{\partial \phi_{2}}{\partial \xi_{1}}\right)+ \\
& +\frac{Q_{66}}{A_{2}}\left(\left(1+\frac{\xi_{3}}{R_{1}}\right) \frac{\partial u}{\partial \xi_{2}}+\xi_{3}\left(y^{*} \frac{\partial \phi_{1}}{\partial \xi_{2}}-\frac{\partial^{2} w}{a_{1} \partial \xi_{1} \partial \xi_{2}}\right)+f\left(\xi_{3}\right) \frac{\partial \phi_{1}}{\partial \xi_{2}}\right) \\
& \sigma_{4}-\mu^{2}\left(\frac{\partial^{2} \sigma_{4}}{a_{1}^{2} \partial \xi_{1}^{2}}+\frac{\partial^{2} \sigma_{4}}{a_{2}^{2} \partial \xi_{2}^{2}}\right)=Q_{44}\left(\frac{1}{A_{2}} \frac{\partial w}{\partial \xi_{2}}+\frac{1}{R_{2}} v+y^{*} \phi_{2}-\frac{\partial w}{a_{2} \partial \xi_{2}}+\frac{d f\left(\xi_{3}\right)}{d \xi_{3}} \phi_{2}\right) \\
& \sigma_{5}-\mu^{2}\left(\frac{\partial^{2} \sigma_{5}}{a_{1}^{2} \partial \xi_{1}^{2}}+\frac{\partial^{2} \sigma_{5}}{a_{2}^{2} \partial \xi_{2}^{2}}\right)=Q_{55}\left(\frac{1}{A_{1}} \frac{\partial w}{\partial \xi_{1}}+\frac{1}{R_{1}} u+y^{*} \phi_{1}-\frac{\partial w}{a_{1} \partial \xi_{1}}+\frac{d f\left(\xi_{3}\right)}{d \xi_{3}} \phi_{1}\right)
\end{aligned}
$$

The equations of motion are derived from the Hamilton's principle, defined in a variational form as follows.

$$
\int_{t_{1}}^{t_{2}}(\delta U-\delta T+\delta W) \mathrm{d} t=0
$$

where $\delta U$ and $\delta T$ denote the variation of the strain energy and kinetic energy, respectively, $\delta W$ is the variation of the external work. Equation (10) can be written in an extended version as follows

$$
\int_{t_{1}}^{t_{2}} \int_{0}^{a} \int_{0}^{b} \int_{-\frac{h}{2}}^{\frac{h}{2}}\left(\sigma_{1} \delta \varepsilon_{1}+\sigma_{2} \delta \varepsilon_{2}+\sigma_{6} \delta \varepsilon_{6}+\sigma_{4} \delta \varepsilon_{4}+\sigma_{5} \delta \varepsilon_{5}\right) \mathrm{a}_{1} \mathrm{a}_{2} \mathrm{a}_{3} \mathrm{~d} \xi_{1} \mathrm{~d} \xi_{2} \mathrm{~d} \xi_{3} \mathrm{~d} t-\int_{-\frac{h}{2}}^{\frac{h}{2}} q \delta w \mathrm{a}_{1} \mathrm{a}_{2} \mathrm{a}_{3} \mathrm{~d} \xi_{1} \mathrm{~d} \xi_{2} \mathrm{~d} \xi_{3} \mathrm{~d} t=0
$$

where $q$ is the transverse load. The variation of the strain energy can be defined in terms of axial, shear, and moment resultants, as follows

$$
\begin{aligned}
& \delta U=\int_{0}^{a} \int_{0}^{b} \int_{-\frac{h}{2}}^{\frac{h}{2}}\left(\sigma_{1} \delta \varepsilon_{1}+\sigma_{2} \delta \varepsilon_{2}+\sigma_{6} \delta \varepsilon_{6}+\sigma_{4} \delta \varepsilon_{4}+\sigma_{5} \delta \varepsilon_{5}\right) \mathrm{a}_{1} \mathrm{a}_{2} \mathrm{a}_{3} \mathrm{~d} \xi_{1} \mathrm{~d} \xi_{2} \mathrm{~d} \xi_{3}= \\
& =\int_{0}^{a} \int_{0}^{b}\left[\frac{N_{1}}{A_{1}}\left(\left(1+\frac{\xi_{3}}{R_{1}}\right) \frac{\partial \delta u}{\partial \xi_{1}}+\frac{1}{a_{2}} \frac{\partial a_{1}}{\partial \xi_{2}}\left(1+\frac{\xi_{3}}{R_{2}}\right) \delta v+\frac{a_{1}}{R_{1}} \delta w\right)+\frac{M_{1}}{A_{1}}\left(y^{*} \frac{\partial \delta \phi_{1}}{\partial \xi_{1}}-\frac{\partial^{2} \delta w}{a_{1} \partial \xi_{1}^{2}}\right)+\frac{P_{1}}{A_{1}}\left(\frac{\partial \delta \phi_{1}}{\partial \xi_{1}}\right)\right] \mathrm{a}_{1} \mathrm{a}_{2} \mathrm{~d} \xi_{1} \mathrm{~d} \xi_{2}+ \\
& +\int_{0}^{a} \int_{0}^{b}\left[\frac{N_{2}}{A_{2}}\left(\left(1+\frac{\xi_{3}}{R_{2}}\right) \frac{\partial \delta v}{\partial \xi_{2}}+\frac{1}{a_{1}} \frac{\partial a_{2}}{\partial \xi_{1}}\left(1+\frac{\xi_{3}}{R_{1}}\right) \delta u+\frac{a_{2}}{R_{2}} \delta w\right)+\frac{M_{2}}{A_{2}}\left(y^{y} \frac{\partial \delta \phi_{2}}{\partial \xi_{2}}-\frac{\partial^{2} \delta w}{a_{2} \partial \xi_{2}^{2}}\right)+\frac{P_{2}}{A_{2}}\left(\frac{\partial \delta \phi_{2}}{\partial \xi_{2}}\right)\right] \mathrm{a}_{1} \mathrm{a}_{2} \mathrm{~d} \xi_{1} \mathrm{~d} \xi_{2}+ \\
& +\int_{0}^{a} \int_{0}^{b}\left[\begin{array}{l}
N_{6}\left(\frac{1}{A_{1}}\left(1+\frac{\xi_{3}}{R_{2}}\right) \frac{\partial \delta v}{\partial \xi_{1}}+\frac{1}{A_{2}}\left(1+\frac{\xi_{3}}{R_{1}}\right) \frac{\partial \delta u}{\partial \xi_{2}}\right)+ \\
+M_{6}\left(\frac{1}{A_{1}}\left(y^{\partial} \frac{\partial \delta \phi_{2}}{\partial \xi_{1}}-\frac{\partial^{2} 2 w}{a_{2} \partial \xi_{1} \partial \xi_{2}}\right)+\frac{1}{A_{2}}\left(y^{*} \frac{\partial \delta \phi_{1}}{\partial \xi_{2}}-\frac{\partial^{2} \delta w}{a_{1} \partial \xi_{1} \partial \xi_{2}}\right)\right)+P_{6}\left(\frac{1}{A_{1}} \frac{\partial \delta \phi_{2}}{\partial \xi_{1}}+\frac{1}{A_{2}} \frac{\partial \delta \phi_{1}}{\partial \xi_{2}}\right)
\end{array}\right] \mathrm{a}_{1} \mathrm{a}_{2} \mathrm{~d} \xi_{1} \mathrm{~d} \xi_{2}+ \\
& +\int_{0}^{a} \int_{0}^{b}\left[Q_{2}\left(\frac{1}{A_{2}} \frac{\partial \delta \xi_{2}}{\partial \xi_{2}}+\frac{1}{R_{2}} \delta v+y^{*} \delta \phi_{2}-\frac{\partial \delta \delta v}{a_{2} \partial \xi_{2}}\right)+K_{2} \delta \phi_{2}\right] \mathrm{a}_{1} \mathrm{a}_{2} \mathrm{~d} \xi_{1} \mathrm{~d} \xi_{2}+ \\
& +\int_{0}^{a} \int_{0}^{b}\left[Q_{1}\left(\frac{1}{A_{1}} \frac{\partial \delta \xi_{1}}{\partial \xi_{1}}+\frac{1}{R_{1}} \delta u+y^{*} \delta \phi_{1}-\frac{\partial \delta \omega v}{a_{1} \partial \xi_{1}}\right)+K_{1} \delta \phi_{1}\right] \mathrm{a}_{1} \mathrm{a}_{2} \mathrm{~d} \xi_{1} \mathrm{~d} \xi_{2}
\end{aligned}
$$


where

$$
\begin{aligned}
& \left(N_{i}, M_{i}, P_{i}\right)=\int_{-\frac{h}{2}}^{\frac{h}{2}} \sigma_{i}\left(1, \xi_{3}, f(\xi)\right) d \xi_{3} \quad(i=1,2,6) \\
& \left(Q_{1}, K_{1}\right)=\int_{-\frac{h}{2}}^{\frac{h}{2}} \sigma_{5}\left(1, \frac{d f}{d \xi}\right) d \xi_{3} \\
& \left(Q_{2}, K_{2}\right)=\int_{-\frac{h}{2}}^{\frac{h}{2}} \sigma_{4}\left(1, \frac{d f}{d \xi}\right) d \xi_{3}
\end{aligned}
$$

By substituting Equation (12) in Equation (11), the static equations for the isotropic doubly-curved nanoshells can be derived by the formula below

$$
\begin{aligned}
& \delta u: \frac{\partial}{\partial \xi_{1}}\left(\frac{N_{1}}{A_{1}}\left(1+\frac{\xi_{3}}{R_{1}}\right)\right)+\frac{\partial}{\partial \xi_{2}}\left(\frac{N_{6}}{A_{2}}\left(1+\frac{\xi_{3}}{R_{1}}\right)\right)-\frac{N_{2}}{A_{2}}\left(\frac{1}{a_{1}} \frac{\partial a_{2}}{\partial \xi_{1}}\left(1+\frac{\xi_{3}}{R_{1}}\right)\right)-\frac{Q_{1}}{R_{1}}=0 \\
& \delta v: \frac{\partial}{\partial \xi_{2}}\left(\frac{N_{2}}{A_{2}}\left(1+\frac{\xi_{3}}{R_{2}}\right)\right)+\frac{\partial}{\partial \xi_{1}}\left(\frac{N_{6}}{A_{1}}\left(1+\frac{\xi_{3}}{R_{1}}\right)\right)-\frac{N_{1}}{A_{1}}\left(\frac{1}{a_{2}} \frac{\partial a_{1}}{\partial \xi_{2}}\left(1+\frac{\xi_{3}}{R_{2}}\right)\right)-\frac{Q_{2}}{R_{2}}=0 \\
& \delta w: \frac{\partial^{2}}{\partial \xi_{1}^{2}}\left(\frac{M_{1}}{A_{1} a_{1}}\right)+\frac{\partial^{2}}{\partial \xi_{2}^{2}}\left(\frac{M_{2}}{A_{2} a_{2}}\right)+\frac{\partial^{2}}{\partial \xi_{1} \partial \xi_{2}}\left(\frac{M_{6}}{A_{1} a_{2}}\right)+\frac{\partial^{2}}{\partial \xi_{1} \partial \xi_{2}}\left(\frac{M_{6}}{A_{2} a_{1}}\right)+\frac{\partial}{\partial \xi_{2}}\left(\frac{Q_{2}}{A_{2}}\right)+\frac{\partial}{\partial \xi_{1}}\left(\frac{Q_{1}}{A_{1}}\right)+ \\
& -\frac{\partial}{\partial \xi_{2}}\left(\frac{Q_{2}}{a_{2}}\right)-\frac{\partial}{\partial \xi_{1}}\left(\frac{Q_{1}}{a_{1}}\right)-\frac{a_{1}}{R_{1}} \frac{N_{1}}{A_{1}}-\frac{a_{2}}{R_{2}} \frac{N_{2}}{A_{2}}=-q \\
& \delta \phi_{1}: \frac{\partial}{\partial \xi_{1}}\left(\frac{M_{1} y^{*}}{A_{1}}\right)+\frac{\partial}{\partial \xi_{2}}\left(\frac{M_{6} y^{*}}{A_{2}}\right)+\frac{\partial}{\partial \xi_{1}}\left(\frac{P_{1}}{A_{1}}\right)+\frac{\partial}{\partial \xi_{2}}\left(\frac{P_{6}}{A_{2}}\right)-Q_{1} y^{*}-K_{1}=0 \\
& \delta \phi_{2}: \frac{\partial}{\partial \xi_{2}}\left(\frac{M_{2} y^{*}}{A_{2}}\right)+\frac{\partial}{\partial \xi_{1}}\left(\frac{M_{6} y^{*}}{A_{1}}\right)+\frac{\partial}{\partial \xi_{2}}\left(\frac{P_{2}}{A_{2}}\right)+\frac{\partial}{\partial \xi_{1}}\left(\frac{P_{6}}{A_{1}}\right)-Q_{2} y^{*}-K_{2}=0
\end{aligned}
$$

By substituting Equations (9) and (13) into Equation (14), the equations of motion for deep spherical panels can be rewritten in terms of displacement components, as shown below

$$
\begin{aligned}
& \frac{A_{11}}{R_{1}} \frac{\partial w}{a_{1} \partial \xi_{1}}+A_{13} \frac{\partial^{2} u}{\partial \xi^{2}}+A_{16}\left(y^{*} \frac{\partial^{2} \phi_{1}}{a_{1}^{2} \partial \xi_{1}^{2}}-\frac{\partial^{3} w}{a_{1}^{3} \partial \xi_{1}^{3}}\right)+A_{17} \frac{\partial^{2} \phi_{1}}{a_{1}^{2} \partial \xi_{1}^{2}}+\frac{B_{11}}{R_{2}} \frac{\partial w}{a_{1} \partial \xi_{1}}+ \\
& +B_{15} \frac{\partial^{2} v}{a_{1} a_{2} \partial \xi_{1} \partial \xi_{2}}+B_{16}\left(y^{*} \frac{\partial^{2} \phi_{2}}{a_{1} a_{2} \partial \xi_{1} \partial \xi_{2}}-\frac{\partial^{3} w}{a_{1} a_{2}^{2} \partial \xi_{1} \partial \xi_{2}^{2}}\right)+B_{17} \frac{\partial^{2} \phi_{2}}{a_{1} a_{2} \partial \xi_{1} \partial \xi_{2}}+ \\
& +D_{13} \frac{\partial^{2} u}{a_{2}^{2} \partial \xi^{2}{ }_{2}}+D_{15} \frac{\partial^{2} v}{a_{1} a_{2} \partial \xi_{1} \partial \xi_{2}}+D_{16}\left(y^{*}\left(\frac{\partial^{2} \phi_{1}}{\partial \xi^{2}{ }_{2}}+\frac{\partial^{2} \phi_{2}}{a_{1} a_{2} \partial \xi_{1} \partial \xi_{2}}\right)-\frac{2 \partial^{3} w}{a_{1} a_{2}^{2} \partial \xi_{1} \partial \xi_{2}^{2}}\right)+ \\
& +D_{17}\left(\frac{\partial^{2} \phi_{2}}{a_{1} a_{2} \partial \xi_{1} \partial \xi_{2}}+\frac{\partial^{2} \phi_{1}}{a_{2}^{2} \partial \xi_{2}^{2}}\right)-\frac{G_{11}}{R_{1}}\left(\frac{\partial w}{a_{1} \partial \xi_{1}}+\frac{1}{R_{1}} u+y^{*} \phi_{1}-\frac{\partial w}{a_{1} \partial \xi_{1}}\right)-\frac{1}{R_{1}} G_{13} \phi_{1}=0 \\
& \frac{B_{12}}{R_{1}} \frac{\partial w}{a_{2} \partial \xi_{2}}+B_{15} \frac{\partial^{2} u}{a_{1} a_{2} \partial \xi_{1} \partial \xi_{2}}+B_{18}\left(y^{*} \frac{\partial^{2} \phi_{1}}{a_{1} a_{2} \partial \xi_{1} \partial \xi_{2}}-\frac{\partial^{3} w}{a_{1}^{2} a_{2} \partial \xi_{1}^{2} \partial \xi_{2}}\right)+B_{19} \frac{\partial^{2} \phi_{1}}{a_{1} a_{2} \partial \xi_{1} \partial \xi_{2}}+ \\
& +\frac{C_{12}}{R_{2}} \frac{\partial w}{a_{2} \partial \xi_{2}}+C_{14} \frac{\partial^{2} v}{a_{2}^{2} \partial \xi_{2}^{2}}+C_{18}\left(y^{*} \frac{\partial^{2} \phi_{2}}{a_{2}^{2} \partial \xi_{2}^{2}}-\frac{\partial^{3} w}{a_{2}^{3} \partial \xi_{2}^{3}}\right)+C_{19} \frac{\partial^{2} \phi_{2}}{a_{2}^{2} \partial \xi_{2}^{2}}+ \\
& +D_{13} \frac{\partial^{2} u}{a_{1} a_{2} \partial \xi_{2} \partial \xi_{1}}+D_{15} \frac{\partial^{2} v}{a_{1}^{2} \partial \xi_{1}{ }^{2}}+D_{16}\left(y^{*}\left(\frac{\partial^{2} \phi_{2}}{a_{1}^{2} \partial \xi^{2} 1}+\frac{\partial^{2} \phi_{1}}{a_{1} a_{2} \partial \xi_{2} \partial \xi_{1}}\right)-\frac{2 \partial^{3} w}{a_{1}^{2} a_{2} \partial \xi_{1} 2 \partial \xi_{2}}\right)+ \\
& +D_{17}\left(\frac{\partial^{2} \phi_{1}}{a_{1} a_{2} \partial \xi_{1} \partial \xi_{2}}+\frac{\partial^{2} \phi_{2}}{a_{1}^{2} \partial \xi_{1}^{2}}\right)-\frac{F_{11}}{R_{2}}\left(\frac{\partial w}{a_{2} \partial \xi_{2}}+\frac{1}{R_{2}} v+y^{*} \phi_{2}-\frac{\partial w}{a_{2} \partial \xi_{2}}\right)-\frac{1}{R_{2}} F_{13} \phi_{2}=0
\end{aligned}
$$




$$
\begin{aligned}
& -\frac{A_{4}}{R_{1}{ }^{2}} w-\frac{A_{5}}{R_{1}}\left(y^{*} \frac{\partial \phi_{1}}{a_{1} \partial \xi_{1}}-\frac{2 \partial^{2} w}{a_{1}^{2} \partial \xi_{1}^{2}}\right)+A_{6}\left(y^{*} \frac{\partial^{3} \phi_{1}}{a_{1}^{3} \partial \xi^{3} 1}-\frac{\partial^{4} w}{a_{1}^{4} \partial \xi_{1}^{4}}\right)-\frac{A_{8}}{R_{1}} \frac{\partial \phi_{1}}{a_{1} \partial \xi_{1}}+A_{9} \frac{\partial^{3} \phi_{1}}{a_{1}^{3} \partial \xi^{3} 1}+ \\
& -\frac{A_{11}}{R_{1}} \frac{\partial u}{a_{1} \partial \xi_{1}}+A_{16} \frac{\partial^{3} u}{a_{1}^{3} \partial \xi^{3}{ }_{1}}-\frac{2 B_{4}}{R_{1} R_{2}} w-B_{5}\left(\frac{1}{R_{1}}\left(y^{*} \frac{\partial \phi_{2}}{a_{2} \partial \xi_{2}}-\frac{2 \partial^{2} w}{a_{2}^{2} \partial \xi_{2}^{2}}\right)+\frac{1}{R_{2}}\left(y^{*} \frac{\partial \phi_{1}}{a_{1} \partial \xi_{1}}-\frac{2 \partial^{2} w}{a_{1}^{2} \partial \xi_{1}^{2}}\right)\right)+ \\
& +B_{6}\left(y^{*}\left(\frac{\partial^{3} \phi_{2}}{a_{1}^{2} a_{2} \partial \xi^{2}{ }_{1} \partial \xi_{2}}+\frac{\partial^{3} \phi_{1}}{a_{2}^{2} a_{1} \partial \xi_{2}{ }^{2} \partial \xi_{1}}\right)-\frac{2 \partial^{4} w}{a_{1}^{2} a_{2}^{2} \partial \xi^{2}{ }_{1} \partial \xi_{2}^{2}}\right)-B_{8}\left(\frac{1}{R_{1}} \frac{\partial \phi_{2}}{a_{2} \partial \xi_{2}}+\frac{1}{R_{2}} \frac{\partial \phi_{1}}{a_{1} \partial \xi_{1}}\right)+ \\
& +B_{9}\left(\frac{\partial^{3} \phi_{1}}{a_{2}^{2} a_{1} \partial \xi_{2}^{2} \partial \xi_{1}}+\frac{\partial^{3} \phi_{2}}{a_{1}^{2} a_{2} \partial \xi^{2}{ }_{1} \partial \xi_{2}}\right)-\frac{B_{11}}{R_{2}} \frac{\partial u}{a_{1} \partial \xi_{1}}-\frac{B_{12}}{R_{1}} \frac{\partial v}{a_{2} \partial \xi_{2}}+B_{16} \frac{\partial^{3} u}{a_{2}^{2} a_{1} \partial \xi_{2}^{2} \partial \xi_{1}}+B_{18} \frac{\partial^{3} v}{a_{1}^{2} a_{2} \partial \xi^{2} \partial \xi_{2}}+ \\
& -\frac{C_{4}}{R_{2}{ }_{2}} w-\frac{C_{5}}{R_{2}}\left(y^{*} \frac{\partial \phi_{2}}{a_{2} \partial \xi_{2}}-\frac{2 \partial^{2} w}{a_{2}^{2} \partial \xi_{2}^{2}}\right)+C_{6}\left(y^{*} \frac{\partial^{3} \phi_{2}}{a_{2}^{3} \partial \xi^{3}{ }_{2}}-\frac{\partial^{4} w}{a_{2}^{4} \partial \xi_{2}{ }^{4}}\right)-\frac{C_{8}}{R_{2}} \frac{\partial \phi_{2}}{a_{2} \partial \xi_{2}}+C_{9} \frac{\partial^{3} \phi_{2}}{a_{2}^{3} \partial \xi^{3}{ }_{2}}-\frac{C_{12}}{R_{2}} \frac{\partial v}{a_{2} \partial \xi_{2}}+C_{18} \frac{\partial^{3} v}{a_{2}^{3} \partial \xi^{3}{ }_{2}}+ \\
& +2 D_{6}\left(y^{*}\left(\frac{\partial^{3} \phi_{1}}{a_{1} a_{2}^{2} \partial \xi_{1} \partial \xi^{2} 2}+\frac{\partial^{3} \phi_{2}}{a_{1}^{2} a_{2} \partial \xi^{2}{ }_{1} \partial \xi_{2}}\right)-\frac{2 \partial^{4} w}{a_{1}^{2} a_{2}^{2} \partial \xi_{1}^{2} \partial \xi^{2}{ }_{2}}\right)+ \\
& +2 D_{9}\left(\frac{\partial^{3} \phi_{1}}{a_{1} a_{2}^{2} \partial \xi^{2} \partial \xi_{1}}+\frac{\partial^{3} \phi_{2}}{a_{1}^{2} a_{2} \partial \xi^{2}{ }_{1} \partial \xi_{2}}\right)+2 D_{16} \frac{\partial^{3} u}{a_{1} a_{2}^{2} \partial \xi_{1} \partial \xi^{2}{ }_{2}}+2 D_{18} \frac{\partial^{3} v}{a_{1}^{2} a_{2} \partial \xi^{2}{ }_{1} \partial \xi_{2}}=\left(1-\mu^{2} \nabla^{2}\right)(-q) \\
& \frac{A_{5} y^{*}}{R_{1}} \frac{\partial w}{a_{1} \partial \xi_{1}}+A_{6} y^{*}\left(y^{*} \frac{\partial^{2} \phi_{1}}{a_{1}^{2} \partial \xi^{2}{ }_{1}}-\frac{\partial^{3} w}{a_{1}^{3} \partial \xi_{1}{ }^{3}}\right)+\frac{A_{8}}{R_{1}} \frac{\partial w}{a_{1} \partial \xi_{1}}+A_{9}\left(2 y^{*} \frac{\partial^{2} \phi_{1}}{a_{1}^{2} \partial \xi^{2}{ }_{1}}-\frac{\partial^{3} w}{a_{1}^{3} \partial \xi_{1}{ }^{3}}\right)+A_{10} \frac{\partial^{2} \phi_{1}}{a_{1}^{2} \partial \xi^{2}{ }_{1}}+ \\
& +A_{16} y^{*} \frac{\partial^{2} u}{a_{1}^{2} \partial \xi^{2} 1}+A_{17} \frac{\partial^{2} u}{a_{1}^{2} \partial \xi_{1}^{2}}+\frac{B_{5} y^{*}}{R_{2}} \frac{\partial w}{a_{1} \partial \xi_{1}}+B_{6} y^{*}\left(y^{*} \frac{\partial^{2} \phi_{2}}{a_{1} a_{2} \partial \xi_{1} \partial \xi_{2}}-\frac{\partial^{3} w}{a_{1} a_{2}^{2} \partial \xi_{1} \partial \xi_{2}^{2}}\right)+\frac{B_{8}}{R_{2}} \frac{\partial w}{a_{1} \partial \xi_{1}}+ \\
& +B_{9}\left(2 y^{*} \frac{\partial^{2} \phi_{2}}{a_{1} a_{2} \partial \xi_{1} \partial \xi_{2}}-\frac{\partial^{3} w}{a_{1} a_{2}^{2} \partial \xi_{1} \partial \xi_{2}{ }^{2}}\right)+B_{10} \frac{\partial^{2} \phi_{2}}{a_{1} a_{2} \partial \xi_{1} \partial \xi_{2}}+B_{18} y^{*} \frac{\partial^{2} v}{a_{1} a_{2} \partial \xi_{1} \partial \xi_{2}}+B_{19} \frac{\partial^{2} v}{a_{1} a_{2} \partial \xi_{1} \partial \xi_{2}}+ \\
& +D_{6} y^{*}\left(y^{*}\left(\frac{\partial^{2} \phi_{1}}{a_{2}^{2} \partial \xi^{2} 2}+\frac{\partial^{2} \phi_{2}}{a_{1} a_{2} \partial \xi_{1} \partial \xi_{2}}\right)-\frac{2 \partial^{3} w}{a_{1} a_{2}^{2} \partial \xi_{1} \partial \xi^{2} 2}\right)+2 D_{9}\left(y^{*}\left(\frac{\partial^{2} \phi_{1}}{a_{2}^{2} \partial \xi^{2} 2}+\frac{\partial^{2} \phi_{2}}{a_{1} a_{2} \partial \xi_{1} \partial \xi_{2}}\right)-\frac{\partial^{3} w}{a_{1} a_{2}^{2} \partial \xi_{1} \partial \xi_{2}^{2}}\right)+ \\
& +D_{10}\left(\frac{\partial^{2} \phi_{1}}{a_{2}^{2} \partial \xi_{2}^{2}}+\frac{\partial^{2} \phi_{2}}{a_{1} a_{2} \partial \xi_{1} \partial \xi_{2}}\right)+D_{16} y^{*} \frac{\partial^{2} u}{a_{2}^{2} \partial \xi^{2} 2}+D_{17} \frac{\partial^{2} u}{a_{2}^{2} \partial \xi^{2} 2}+D_{18} y^{*} \frac{\partial^{2} v}{a_{1} a_{2} \partial \xi_{1} \partial \xi_{2}}+D_{19} \frac{\partial^{2} v}{a_{1} a_{2} \partial \xi_{1} \partial \xi_{2}}+ \\
& -G_{11} y^{*}\left(\frac{1}{R_{1}} u+y^{*} \phi_{1}\right)-G_{13}\left(\frac{1}{R_{1}} u+2 y^{*} \phi_{1}\right)-G_{14} \phi_{1}=0 \\
& \frac{B_{5} y^{*}}{R_{1}} \frac{\partial w}{a_{2} \partial \xi_{2}}+B_{6} y^{*}\left(y^{*} \frac{\partial^{2} \phi_{1}}{a_{1} a_{2} \partial \xi_{1} \partial \xi_{2}}-\frac{\partial^{3} w}{a_{1}^{2} a_{2} \partial \xi_{1}^{2} \partial \xi_{2}}\right)+\frac{B_{8}}{R_{1}} \frac{\partial w}{a_{2} \partial \xi_{2}}+B_{9}\left(2 y^{*} \frac{\partial^{2} \phi_{1}}{a_{1} a_{2} \partial \xi_{1} \partial \xi_{2}}-\frac{\partial^{3} w}{a_{1}^{2} a_{2} \partial \xi_{1}{ }^{2} \partial \xi_{2}}\right)+ \\
& +B_{10} \frac{\partial^{2} \phi_{1}}{a_{1} a_{2} \partial \xi_{1} \partial \xi_{2}}+B_{16} y^{*} \frac{\partial^{2} u}{a_{1} a_{2} \partial \xi_{1} \partial \xi_{2}}+B_{17} \frac{\partial^{2} u}{a_{1} a_{2} \partial \xi_{1} \partial \xi_{2}}+\frac{C_{5} y^{*}}{R_{2}} \frac{\partial w}{a_{2} \partial \xi_{2}}+C_{6} y^{*}\left(y^{*} \frac{\partial^{2} \phi_{2}}{a_{2}^{2} \partial \xi^{2} 2}-\frac{\partial^{3} w}{a_{2}^{3} \partial \xi_{2}{ }^{3}}\right)+ \\
& +\frac{C_{8}}{R_{2}} \frac{\partial w}{a_{2} \partial \xi_{2}}+C_{9}\left(2 y^{*} \frac{\partial^{2} \phi_{2}}{a_{2}^{2} \partial \xi^{2}{ }_{2}}-\frac{\partial^{3} w}{a_{2}^{3} \partial \xi_{2}{ }^{3}}\right)+C_{10} \frac{\partial^{2} \phi_{2}}{a_{2}^{2} \partial \xi^{2} 2}+C_{18} y^{*} \frac{\partial^{2} v}{a_{2}^{2} \partial \xi^{2}{ }_{2}}+C_{19} \frac{\partial^{2} v}{a_{2}^{2} \partial \xi^{2}{ }_{2}}+ \\
& +D_{6} y^{*}\left(y^{*}\left(\frac{\partial^{2} \phi_{1}}{a_{1} a_{2} \partial \xi_{1} \partial \xi_{2}}+\frac{\partial^{2} \phi_{2}}{a_{1}^{2} \partial \xi^{2}{ }_{1}}\right)-\frac{2 \partial^{3} w}{a_{1}^{2} a_{2} \partial \xi^{2}{ }_{1} \partial \xi_{2}}\right)+2 D_{9}\left(y^{*}\left(\frac{\partial^{2} \phi_{1}}{a_{1} a_{2} \partial \xi_{1} \partial \xi_{2}}+\frac{\partial^{2} \phi_{2}}{a_{1}^{2} \partial \xi^{2}{ }_{1}}\right)-\frac{\partial^{3} w}{a_{1}^{2} a_{2} \partial \xi^{2}{ }_{1} \partial \xi_{2}}\right)+ \\
& +D_{10}\left(\frac{\partial^{2} \phi_{1}}{a_{1} a_{2} \partial \xi_{1} \partial \xi_{2}}+\frac{\partial^{2} \phi_{2}}{a_{1}^{2} \partial \xi^{2}{ }_{1}}\right)+D_{16} y^{*} \frac{\partial^{2} u}{a_{1} a_{2} \partial \xi_{2} \partial \xi_{1}}+D_{17} \frac{\partial^{2} u}{a_{1} a_{2} \partial \xi_{2} \partial \xi_{1}}+D_{18} y^{*} \frac{\partial^{2} v}{a_{1}^{2} \partial \xi^{2}{ }_{1}}+ \\
& +D_{19} \frac{\partial^{2} v}{a_{1}^{2} \partial \xi^{2} 1}-F_{11} y^{*}\left(\frac{1}{R_{2}} v+y^{*} \phi_{2}\right)-F_{13}\left(\frac{1}{R_{2}} v+2 y^{*} \phi_{2}\right)-F_{14} \phi_{2}=0
\end{aligned}
$$

More details about the parameters of Equation (15) are provided in Appendix A. The mathematical background defined in this case has been implemented and solved numerically by using a Navier type procedure, as specified in the following.

\section{Solution Procedure}

Since the exact solution of the partial differential Equation (15) determined in Section 2, for general boundary conditions is difficult, a Navier-type solution is applied for simply-supported doubly-curved nanoshells in this scenario. Thus, the equations related to boundary conditions are defined by the equation below.

$$
\begin{aligned}
& u\left(\xi_{1}, 0\right)=u\left(\xi_{1}, b\right)=v\left(0, \xi_{2}\right)=v\left(a, \xi_{2}\right)=0 \\
& w\left(\xi_{1}, 0\right)=w\left(\xi_{1}, b\right)=w\left(0, \xi_{2}\right)=w\left(a, \xi_{2}\right)=0 \\
& \phi_{1}\left(\xi_{1}, 0\right)=\phi_{1}\left(\xi_{1}, b\right)=\phi_{2}\left(0, \xi_{2}\right)=\phi_{2}\left(a, \xi_{2}\right)=0
\end{aligned}
$$

Based on Equation (16), the solution functions to the partial differential Equation (15) can be expressed by the equation below $[54,55]$. 


$$
\begin{aligned}
& u\left(\xi_{1}, \xi_{2} ; t\right)=\sum_{r=1}^{\infty} \sum_{s=1}^{\infty} U_{r s} \cos \left(\alpha \xi_{1}\right) \sin \left(\beta \xi_{2}\right) \\
& v\left(\xi_{1}, \xi_{2} ; t\right)=\sum_{r=1}^{\infty} \sum_{s=1}^{\infty} V_{r s} \sin \left(\alpha \xi_{1}\right) \cos \left(\beta \xi_{2}\right) \\
& w\left(\xi_{1}, \xi_{2} ; t\right)=\sum_{r=1}^{\infty} \sum_{s=1}^{\infty} W_{r s} \sin \left(\alpha \xi_{1}\right) \sin \left(\beta \xi_{2}\right) \\
& \phi_{1}\left(\xi_{1}, \xi_{2} ; t\right)=\sum_{r=1}^{\infty} \sum_{s=1}^{\infty} \phi_{r s}^{1} \cos \left(\alpha \xi_{1}\right) \sin \left(\beta \xi_{2}\right) \\
& \phi_{2}\left(\xi_{1}, \xi_{2} ; t\right)=\sum_{r=1}^{\infty} \sum_{s=1}^{\infty} \phi_{r s}^{2} \sin \left(\alpha \xi_{1}\right) \cos \left(\beta \xi_{2}\right)
\end{aligned}
$$

where

$$
\alpha=\frac{r \pi}{a}, \beta=\frac{s \pi}{b}
$$

Substituting Equation (17) into Equation (15), the following equations are derived.

$$
\left[K_{i j}\right]\left\{\begin{array}{c}
U_{r s} \\
V_{r s} \\
W_{r s} \\
\phi_{r s}^{1} \\
\phi_{r s}^{2}
\end{array}\right\}=\left\{\begin{array}{c}
0 \\
0 \\
Q_{r s} \\
0 \\
0
\end{array}\right\}, \quad(i, j=1,2, . ., 5)
$$

where $U_{r s}, V_{r s}, W_{r s}, \phi_{r s}^{1}$, and $\phi_{r s}^{2}$ are the unknown coefficients. $\left[K_{i j}\right]$ is the stiffness matrix, whose additional details are provided in Appendix B. $Q_{r s}$ are the coefficients in the double Fourier expansion related to the transverse load, i.e.,

$$
q(x, y)=\sum_{r=1}^{\infty} \sum_{s=1}^{\infty} Q_{r s} \sin \left(\alpha x_{1}\right) \sin \left(\beta x_{2}\right)
$$

\section{Numerical Results and Discussion}

This section is devoted, first, to validate the proposed theory, and then, to evaluate the sensitivity of the static response of symmetric doubly-curved nanoshells by means of a systematic study. The results obtained in the following are divided in two categories, namely, shallow and deep nanoshells. The governing equations of the problem, defined by Equation (19), in compact form, together with its stiffness matrix detailed in Appendix B, are implemented and solved in a MATLAB subroutine in this scenario. The effect of several parameters, such as the nonlocal parameter, the mechanical and geometrical properties, is, thus, investigated on the bending deflection of isotropic doubly-curved shallow and deep nanoshells, while comparing their final response.

\subsection{Comparison and Validation}

This section is devoted to the validation and parametric study of the static behavior of doubly-curved nanoshells. For validation purposes, we determine the deflection and stress response in dimensionless form in agreement with References [56-60], as follows.

$$
\begin{array}{ll}
\bar{w}=w\left(\frac{a}{2}, \frac{b}{2}, 0\right) \frac{10^{2} E h^{3}}{q_{0} a^{4}}, & \bar{\sigma}_{x x}=\sigma_{x x}\left(\frac{a}{2}, \frac{b}{2}, \frac{h}{2}\right) \frac{h^{2}}{q_{0} a^{2}} \\
\bar{\tau}_{x y}=\tau_{x y}\left(0,0, \frac{h}{2}\right) \frac{h^{2}}{q_{0} a^{2}}, & \bar{\tau}_{x z}=\tau_{x z}\left(0, \frac{b}{2}, 0\right) \frac{h}{q_{0} a}, \quad \bar{z}=\frac{z}{h} \times 10
\end{array}
$$

The deflection response is evaluated comparatively between a nonlocal and local theory. Table 1 summarizes the deflection and stress results for square plates under a uniform load, and different side-to-thickness ratios $a / h$. This example represents the limit case for a doubly-curved nanoshell, 
when the curvature radii tends to infinite values $\left(R_{1}=R_{2} \rightarrow \infty\right)$. The numerical results based on our proposed formulation are in line with predictions by Reddy [56,57], Ferreira et al. [58,59], and Xiang et al. [60], where the proposed HSDT yield results in terms of deflection and a stress response. As notable in Table 1 , an increased $a / h$ ratio enables a general decrease in the non-dimensional deflection of the plate, and an overall increase in the stress value, which is in agreement with findings by References [56-60]. A similar parametric study is repeated for symmetric doubly-curved shells under a sinusoidal lateral loading, as summarized in Table 2 . The results are successfully verified against predictions based on a parabolic shear deformation theory (PSDT) and classical thin shell theory (CST), as shown in Reference [61]. Based on results in Table 2, it is visible that an increased $R / a$ ratio enables a general increase in the non-dimensional deflection of the doubly-curved shell, for each fixed value for $h / a$ ratio, whereby, an increased $h / a$ ratio decreases the overall structural deflection due to the increased stiffness of the curved shells. The accuracy of our proposed theory against the available literature [61] is confirmed once again by good agreement between results in Table 2 .

Table 1. Non-dimensional deflection and stress state for square isotropic plates under a uniform load.

\begin{tabular}{|c|c|c|c|}
\hline$\frac{a}{h}$ & Method & $\bar{w}$ & $\bar{\sigma}_{x x}$ \\
\hline \multirow{6}{*}{10} & Exact [56] & 4.791 & 0.2762 \\
\hline & Reddy [57] & 4.77 & 0.2899 \\
\hline & Ferreira et al. [58] & 4.787 & 0.2739 \\
\hline & Ferreira et al. [59] & 4.788 & 0.2762 \\
\hline & Xiang et al. [60] & 4.609 & 0.288 \\
\hline & present & 4.758 & 0.3193 \\
\hline \multirow{6}{*}{20} & Exact [56] & 4.625 & 0.2762 \\
\hline & Reddy [57] & 4.57 & 0.2683 \\
\hline & Ferreira et al. [58] & 4.613 & 0.2737 \\
\hline & Ferreira et al. [59] & 4.616 & 0.2749 \\
\hline & Xiang et al. [60] & 4.442 & 0.276 \\
\hline & present & 4.587 & 0.32 \\
\hline \multirow{6}{*}{50} & Exact [56] & 4.579 & 0.2762 \\
\hline & Reddy [57] & 4.496 & 0.2667 \\
\hline & Ferreira et al. [58] & 4.575 & 0.2787 \\
\hline & Ferreira et al. [59] & 4.578 & 0.2745 \\
\hline & Xiang et al. [60] & 4.396 & 0.284 \\
\hline & present & 4.55 & 0.3203 \\
\hline \multirow{6}{*}{100} & Exact [56] & 4.572 & 0.2762 \\
\hline & Reddy [57] & 4.482 & 0.2664 \\
\hline & Ferreira et al. [58] & 4.573 & 0.2844 \\
\hline & Ferreira et al. [59] & 4.5715 & 0.2744 \\
\hline & Xiang et al. [60] & - & 0.282 \\
\hline & present & 4.5455 & 0.3203 \\
\hline
\end{tabular}


Table 2. Non-dimensional deflection of doubly-curved shallow shells under a sinusoidal distribution of the lateral loading.

\begin{tabular}{|c|c|c|c|c|}
\hline$R / a$ & Method & $h / a=0.01$ & $h / a=0.1$ & $h / a=0.15$ \\
\hline \multirow{4}{*}{1} & Present & 98.1142 & 7.4312 & 3.877 \\
\hline & 3-D [61] & 100.59 & 8.7095 & 4.9497 \\
\hline & PSDT [61] & 99.645 & 7.4751 & 3.8929 \\
\hline & CST [61] & 99.644 & 7.3702 & 3.6979 \\
\hline \multirow{4}{*}{2} & Present & 392.8533 & 16.9774 & 6.8716 \\
\hline & 3-D [61] & 396.45 & 18.451 & 7.724 \\
\hline & PSDT [61] & 394.37 & 17.013 & 6.9261 \\
\hline & CST [61] & 394.37 & 16.48 & 6.3322 \\
\hline \multirow{4}{*}{3} & Present & 870.5856 & 22.1878 & 8.0018 \\
\hline & 3-D [61] & 875.36 & 23.381 & 8.5912 \\
\hline & PSDT [61] & 872.02 & 22.277 & 8.094 \\
\hline & CST [61] & 872 & 21.371 & 7.2945 \\
\hline \multirow{4}{*}{4} & Present & 1512.3 & 24.8495 & 8.4892 \\
\hline & 3-D [61] & 1518.3 & 25.785 & 8.9235 \\
\hline & PSDT [61] & 1513.6 & 24.983 & 8.6017 \\
\hline & CST [61] & 1513.6 & 23.849 & 7.7043 \\
\hline \multirow{4}{*}{5} & Present & 2294.2 & 26.3088 & 8.7352 \\
\hline & 3-D [61] & 2301.4 & 27.061 & 9.0755 \\
\hline & PSDT [61] & 2295.4 & 26.471 & 8.8589 \\
\hline & CST [61] & 2295.3 & 25.201 & 7.9099 \\
\hline \multirow{4}{*}{10} & Present & 7370.9 & 28.5417 & 9.0861 \\
\hline & 3-D [61] & 7383.1 & 28.91 & 9.2502 \\
\hline & PSDT [61] & 7371.3 & 28.754 & 9.2267 \\
\hline & CST [61] & 7370.2 & 27.262 & 8.2019 \\
\hline \multirow{4}{*}{20} & Present & 16,485 & 29.1602 & 9.1782 \\
\hline & 3-D [61] & 16,499 & 29.356 & 9.2666 \\
\hline & PSDT [61] & 16,485 & 29.388 & 9.3235 \\
\hline & CST [61] & 16,479 & 27.831 & 8.2783 \\
\hline \multirow{4}{*}{$\infty$} & Present & 28,039 & 29.3723 & 9.2094 \\
\hline & 3-D [61] & 29,504 & 29.44 & 9.2352 \\
\hline & PSDT [61] & 28,041 & 29.606 & 9.3562 \\
\hline & CST [61] & 28,026 & 28.026 & 8.304 \\
\hline
\end{tabular}

\subsection{Static Analysis of Doubly-Curved Thin Nanoshells}

This subsection studies the mechanical behavior of doubly-curved thin nanoshells, and its sensitivity to the nonlocal and geometrical properties of the nanostructures.

In Figure $2 \mathrm{a}-\mathrm{c}$, we present the evolution of the stress components in a non-dimensional form, throughout the thickness for a thin spherical panel with different nonlocal parameters. Based on these figures, it is worth observing that the stiffness decreases for an increasing nonlocal parameter, along with a general decrease in the natural frequency of the nanostructure, and an increase in the stress components. More specifically, the nonlocal parameter affects the axial and longitudinal shear stress more significantly near the top and bottom sides of the panel, while assuming a null value at the mid-plane, independently of the nonlocal parameter (Figure 2a,b). The contrary occurs for the shear stress component, whose value remains unaltered and equal to zero at both extremity sides, and reaches the peak value at the mid-plane with an increasing magnitude for an increased nonlocal parameter (Figure 2c). 


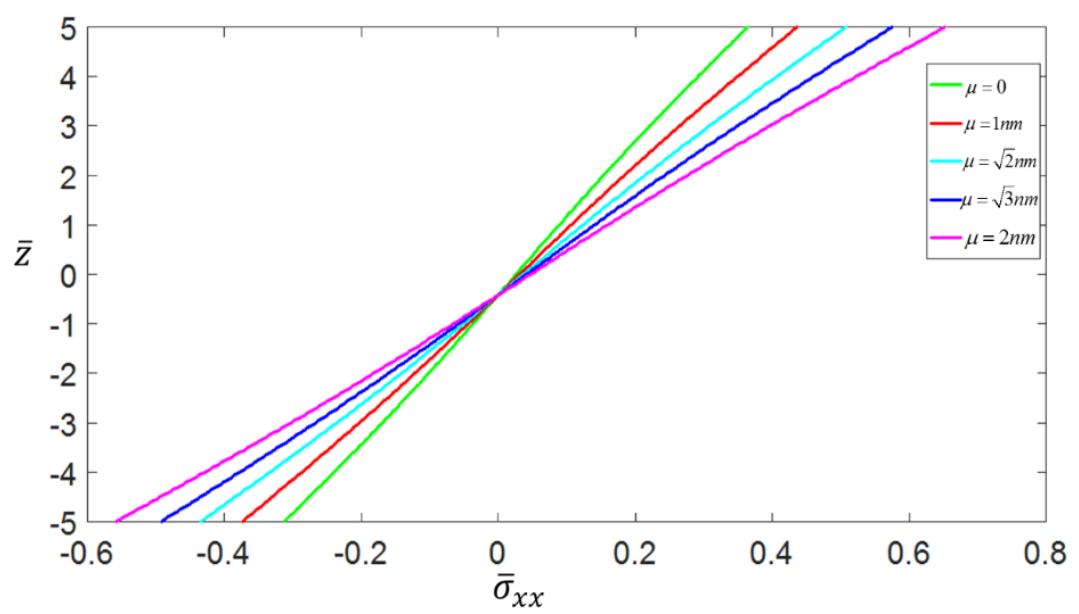

(a)

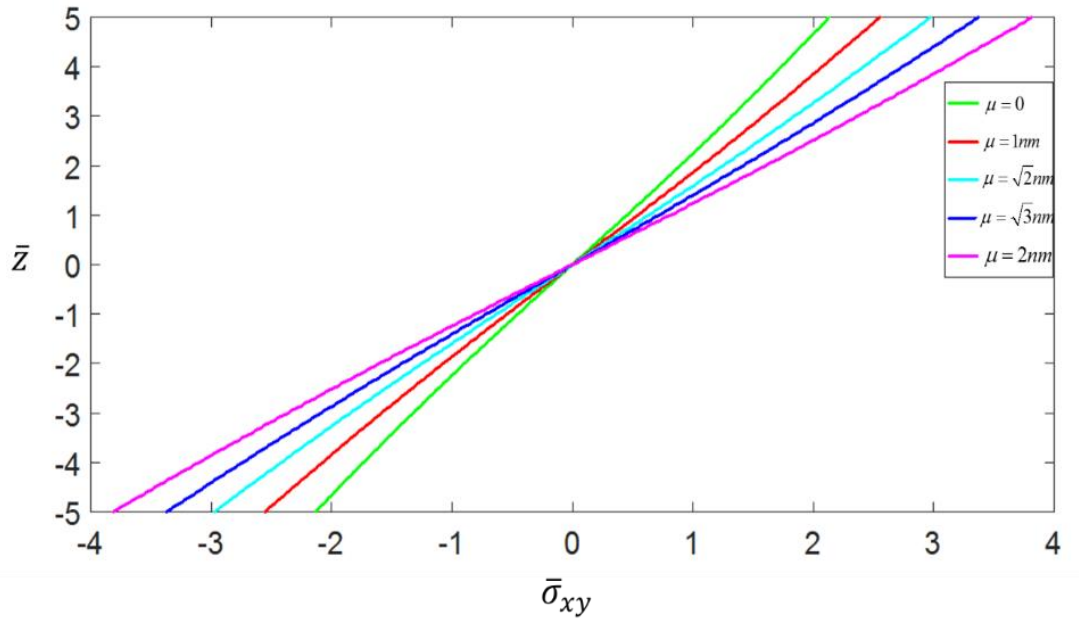

(b)

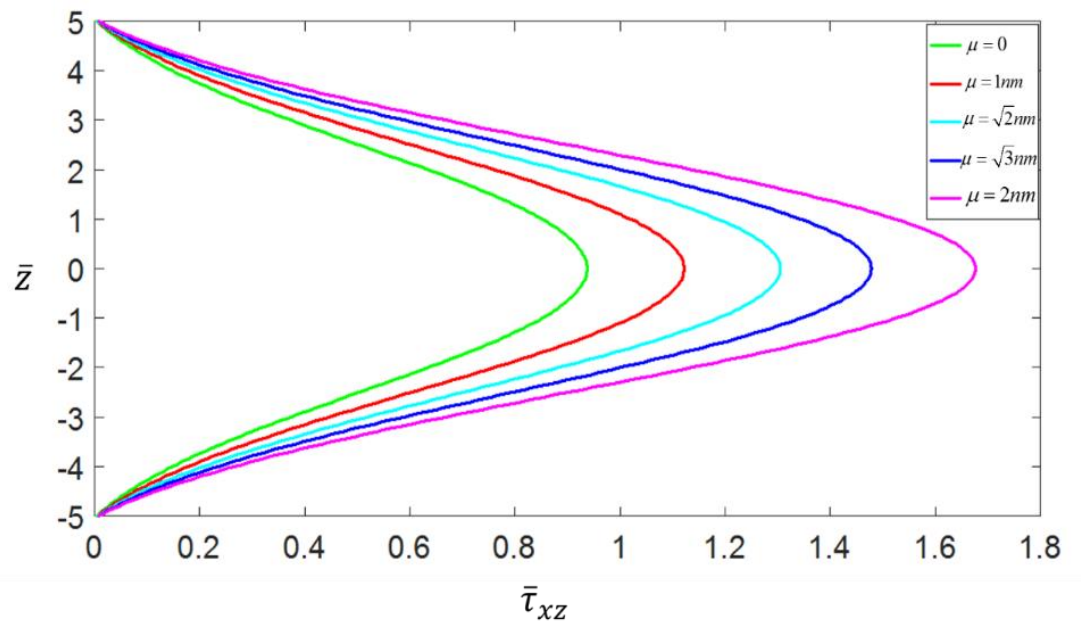

(c)

Figure 2. Variation with the nonlocal parameter of the dimensionless axial stress (a), longitudinal shear stress (b), and transverse shear stress (c) through the thickness of shallow spherical panels with $R_{1}=R_{2}, R / a=10, a / h=10$.

Figure 3 also shows the combined effect of the nonlocal parameter, $\mu$, and side-to-thickness ratio, $a / h$, on the deflection response of thin spherical (Figure 3a) and cylindrical (Figure 3b) panels. In both cases, an increased non-local parameter clearly yields a monotonic increase in deflection for each 
fixed $a / h$ ratio. The deflection also increases for a decreased geometrical $a / h$ ratio, while keeping the nonlocal parameter fixed due to an overall stiffness reduction. Based on a comparative evaluation of the curves in Figure 3a,b under the same assumptions for $\mu$ and $a / h$, the spherical panels seem to be more flexible than the cylindrical panels due to the higher deflections registered for the first geometry. This is in line with the well-known size-dependence of the mechanical properties for small-scaled structures and nanoelectromechanical systems (NEMS), as largely observed in many experimental investigations and atomistic simulations in literature.

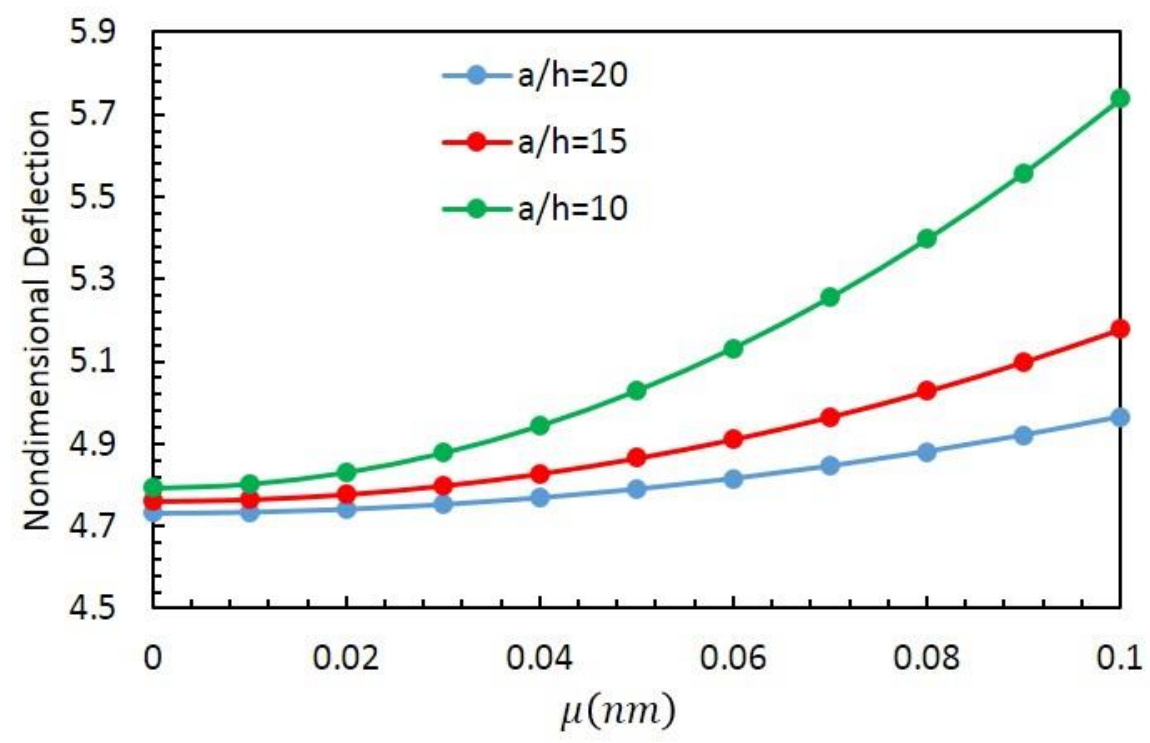

(a)

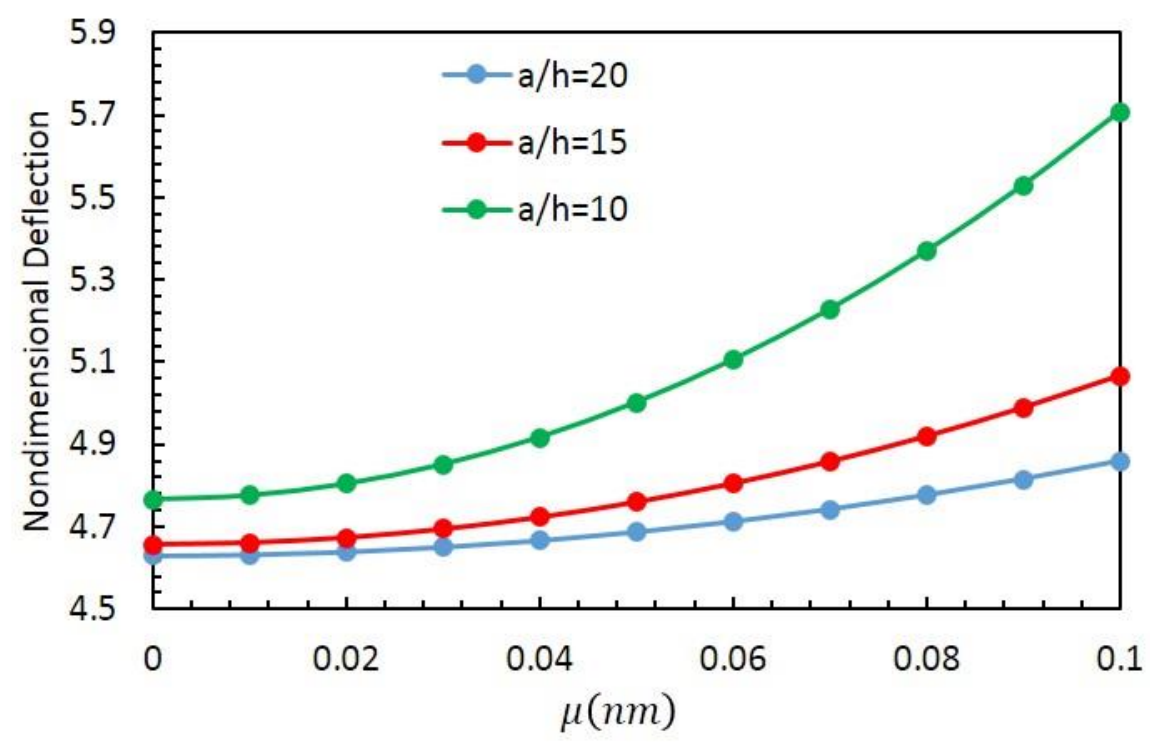

(b)

Figure 3. Effect of the nonlocal parameter and $a / h$ ratio on the deflection of shallow (a) spherical panels with $R_{1}=R_{2}, R / a=20$, and (b) cylindrical panels with $R_{1} / a=20, R_{2} \rightarrow \infty$.

In Figure 4a,b, we plot the effect of the nonlocal parameter and Poisson's ratio on the deflection of the shallow spherical panel and cylindrical panel, respectively, under a uniform load. Both figures clearly show that the structural deflection increases with the nonlocal parameter under a fixed Poisson's ratio, whereby an increased value of the Poisson's ratio reduces the structural deformability under a fixed nonlocal parameter. The double effect of the nonlocal parameter and length-to-side ratio $b / a$ 
is also considered in Figure 5a,b, for a shallow spherical or cylindrical panel, respectively, whose deflection seems to increase for an increased nonlocal parameter and length-to-side ratio. By comparing Figure $5 a, b$, it is worth noticing the higher deformability of spherical panels compared to cylindrical panels under the same assumptions of the $\mu$ and $b / a$ ratio. This variation in stiffness is simply related to topological reasons, which is more pronounced for increasing the ratios $b / a$, as visible in the plots of Figure 5a,b.

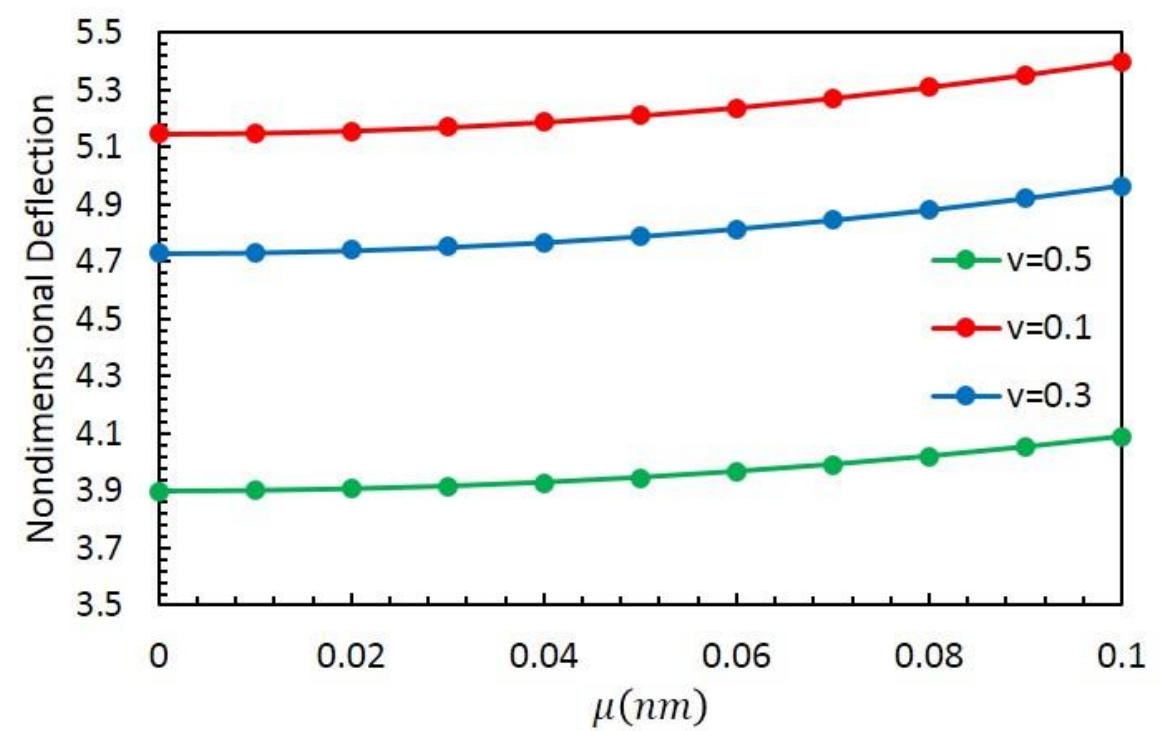

(a)

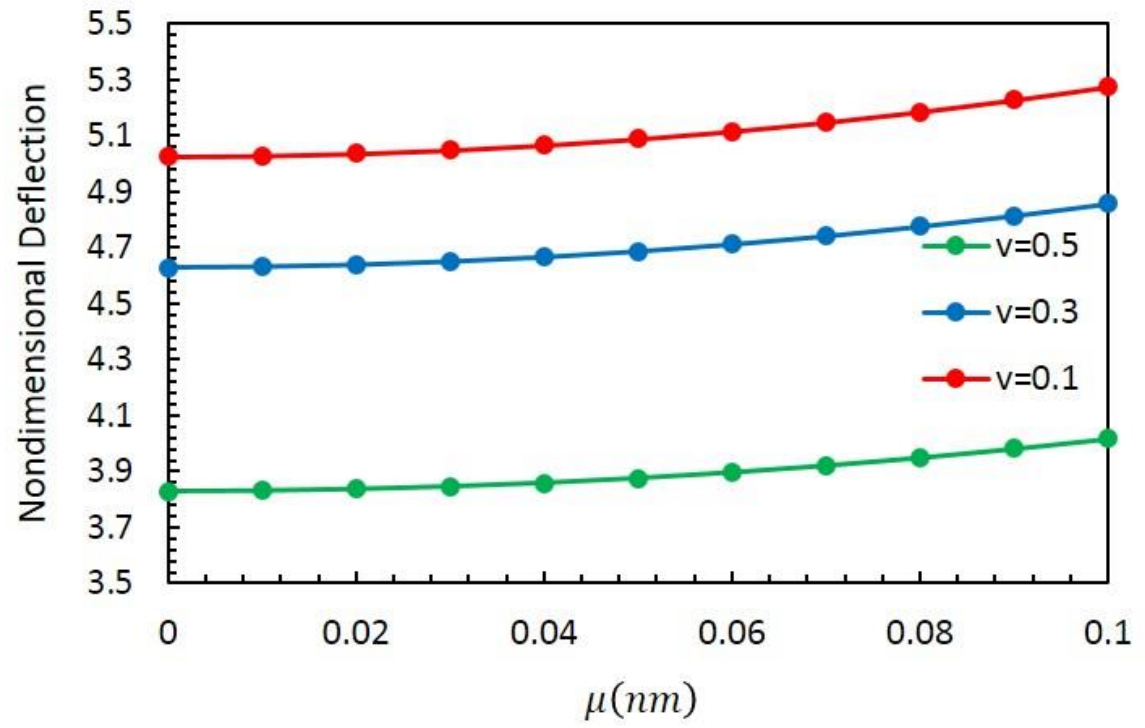

(b)

Figure 4. Effect of the nonlocal parameter and Poisson's coefficient on the deflection of shallow (a) spherical panels with $R_{1}=R_{2}, R / a=20, a / h=20$, (b) cylindrical panels with $R_{1} / a=20, R_{2} \rightarrow \infty, a / h=20$. 


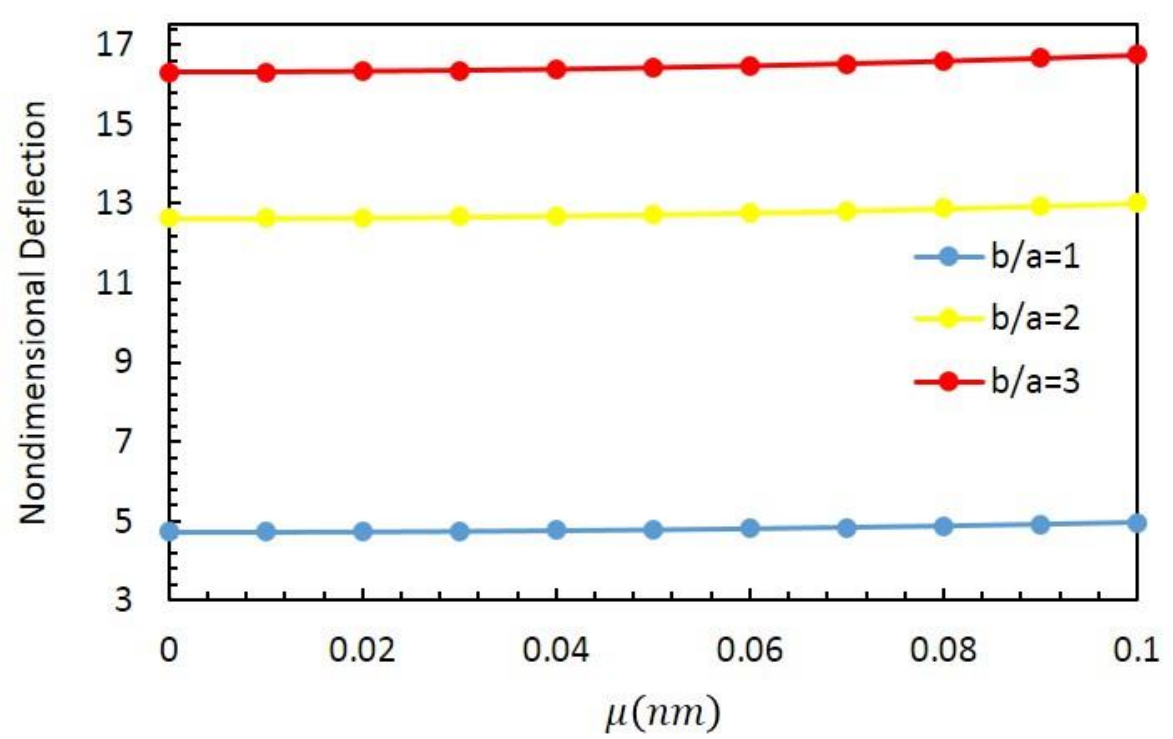

(a)

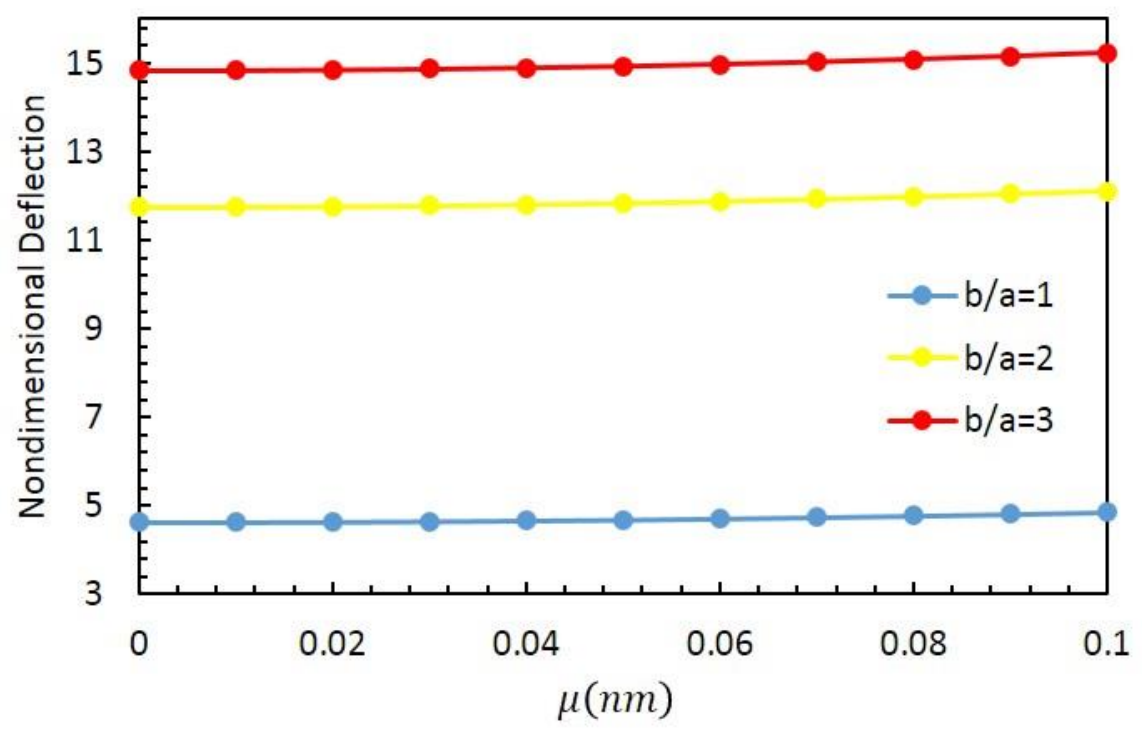

(b)

Figure 5. Effect of the nonlocal parameter and $b / a$ ratio on the deflection of shallow (a) spherical panels with $R_{1}=R_{2}, R / a=20$, and (b) cylindrical panels with $R_{1} / a=20, R_{2} \rightarrow \infty$.

Tables 3 and 4 summarize the results in terms of a deflection response for a thin $(a / h=20)$ and moderately thick $(a / h=10)$ shallow spherical (Table 3$)$ and cylindrical (Table 4$)$ panel, under a uniform load, with different nonlocal parameters, side-to-thickness ratios $(R / a)$, and length-to-side ratios $(b / a)$. As visible in both tables, the deflection response of shallow panels increases for an increased nonlocal parameter as well as for a decreased $R / a$ ratio and an increased $b / a$ ratio due to a global decreased structural stiffness. From a physical standpoint, these results would confirm the importance of a correct definition of nonlocality parameters within nanostructures by means of appropriate experimental tests for different geometries, which could considerably affect the global structural stiffness and functionality of nanosystems. 
Table 3. Non-dimensional deflection of shallow spherical panels $\left(\widetilde{w}=w\left(10^{2} E h^{3}\right) /\left(q_{0} a^{4}\right)\right)$ under a uniform load.

\begin{tabular}{|c|c|c|c|c|c|c|c|}
\hline \multirow{2}{*}{$\mu$} & \multirow{2}{*}{$R / a$} & \multicolumn{2}{|c|}{$b / a=1$} & \multicolumn{2}{|c|}{$b / a=2$} & \multicolumn{2}{|c|}{$b / a=3$} \\
\hline & & $a / h=10$ & $a / h=20$ & $a / h=10$ & $a / h=20$ & $a / h=10$ & $a / h=20$ \\
\hline \multirow{6}{*}{0.25} & 5 & 12.0386 & 11.0094 & 30.0962 & 28.7524 & 40.5842 & 38.0363 \\
\hline & 10 & 10.9468 & 6.7840 & 22.8961 & 19.6583 & 28.0741 & 27.3571 \\
\hline & 20 & 10.7041 & 6.1901 & 21.6039 & 15.0639 & 26.0654 & 19.1014 \\
\hline & 50 & 10.6381 & 6.0420 & 21.2679 & 14.1387 & 25.5534 & 17.6132 \\
\hline & 100 & 10.6287 & 6.0214 & 21.2207 & 14.0157 & 25.4819 & 17.4193 \\
\hline & plate & 10.6256 & 6.0146 & 21.2050 & 13.9752 & 25.4582 & 17.3556 \\
\hline \multirow{6}{*}{0.5} & 5 & 31.9859 & 18.7949 & 69.4048 & 42.6926 & 90.0968 & 54.7284 \\
\hline & 10 & 29.0851 & 11.5815 & 52.8006 & 29.1893 & 62.3243 & 39.3627 \\
\hline & 20 & 28.4402 & 10.5675 & 49.8209 & 22.3674 & 57.8650 & 27.4840 \\
\hline & 50 & 28.2648 & 10.3147 & 49.0459 & 20.9936 & 56.7285 & 25.3427 \\
\hline & 100 & 28.2399 & 10.2796 & 48.9371 & 20.811 & 56.5698 & 25.0637 \\
\hline & plate & 28.2316 & 10.2679 & 48.9010 & 20.7508 & 56.5171 & 24.9721 \\
\hline \multirow{6}{*}{0.75} & 5 & 65.2313 & 31.7707 & 134.9192 & 65.9262 & 172.6177 & 82.5485 \\
\hline & 10 & 59.3154 & 19.5772 & 102.6416 & 45.0744 & 119.4080 & 59.3719 \\
\hline & 20 & 58.0004 & 17.8633 & 96.8491 & 34.540 & 110.8644 & 41.4551 \\
\hline & 50 & 57.6426 & 17.4359 & 95.3426 & 32.4185 & 108.6870 & 38.2252 \\
\hline & 100 & 57.5918 & 17.3765 & 95.1312 & 32.1365 & 108.3829 & 37.8044 \\
\hline & plate & 57.5749 & 17.3567 & 95.0609 & 32.0436 & 108.2819 & 37.6662 \\
\hline \multirow{6}{*}{1} & 5 & 111.7749 & 49.9368 & 226.6394 & 98.4534 & 288.1471 & 121.4968 \\
\hline & 10 & 101.6379 & 30.7713 & 172.4189 & 67.3136 & 199.3252 & 87.3849 \\
\hline & 20 & 99.3836 & 28.0773 & 162.6887 & 51.5815 & 185.0636 & 61.0145 \\
\hline & 50 & 98.7715 & 27.4055 & 160.1579 & 48.4134 & 181.4289 & 56.2606 \\
\hline & 100 & 98.6845 & 27.3121 & 159.8028 & 47.9923 & 180.9213 & 55.6413 \\
\hline & plate & 98.6555 & 27.2811 & 159.6848 & 47.8535 & 180.7527 & 55.4379 \\
\hline
\end{tabular}

Table 4. Non-dimensional deflection of shallow cylindrical panels $\left(\widetilde{w}=w\left(10^{2} E h^{3}\right) /\left(q_{0} a^{4}\right)\right)$ under a uniform load.

\begin{tabular}{|c|c|c|c|c|c|c|c|}
\hline \multirow{2}{*}{$\mu$} & \multirow{2}{*}{$R_{1} / a$} & \multicolumn{2}{|c|}{$b / a=1$} & \multicolumn{2}{|c|}{$b / a=2$} & \multicolumn{2}{|c|}{$b / a=3$} \\
\hline & & $a / h=10$ & $a / h=20$ & $a / h=10$ & $a / h=20$ & $a / h=10$ & $a / h=20$ \\
\hline \multirow{6}{*}{0.25} & 5 & 10.9468 & 6.784 & 21.4586 & 14.6529 & 25.5534 & 17.6132 \\
\hline & 10 & 10.7041 & 6.1901 & 21.2679 & 14.1387 & 25.4819 & 17.4193 \\
\hline & 20 & 10.6451 & 6.0575 & 21.2207 & 14.0157 & 25.4641 & 17.3715 \\
\hline & 50 & 10.6287 & 6.0214 & 21.2076 & 13.9816 & 25.4592 & 17.3582 \\
\hline & 100 & 10.6264 & 6.0163 & 21.2057 & 13.9768 & 25.4584 & 17.3563 \\
\hline & plate & 10.6256 & 6.0146 & 21.205 & 13.9752 & 25.4582 & 17.3556 \\
\hline \multirow{6}{*}{0.5} & 5 & 29.0851 & 11.5815 & 49.4858 & 21.7572 & 56.7285 & 25.3427 \\
\hline & 10 & 28.4402 & 10.5675 & 49.0459 & 20.9936 & 56.5698 & 25.0637 \\
\hline & 20 & 28.2835 & 10.3412 & 48.9371 & 20.811 & 56.5303 & 24.9949 \\
\hline & 50 & 28.2399 & 10.2796 & 48.9068 & 20.7604 & 56.5192 & 24.9757 \\
\hline & 100 & 28.2337 & 10.2708 & 48.9024 & 20.7532 & 56.5176 & 24.973 \\
\hline & plate & 28.2316 & 10.2679 & 48.901 & 20.7508 & 56.5171 & 24.9721 \\
\hline \multirow{6}{*}{0.75} & 5 & 59.3154 & 19.5772 & 96.1977 & 33.5977 & 108.687 & 38.2252 \\
\hline & 10 & 58.0004 & 17.8633 & 95.3426 & 32.4185 & 108.3829 & 37.8044 \\
\hline & 20 & 57.6807 & 17.4807 & 95.1312 & 32.1365 & 108.3072 & 37.7006 \\
\hline & 50 & 57.5918 & 17.3765 & 95.0721 & 32.0584 & 108.286 & 37.6717 \\
\hline & 100 & 57.5791 & 17.3617 & 95.0637 & 32.0473 & 108.2829 & 37.6675 \\
\hline & plate & 57.5749 & 17.3567 & 95.0609 & 32.0436 & 108.2819 & 37.6662 \\
\hline \multirow{6}{*}{1} & 5 & 101.6379 & 30.7713 & 161.5943 & 50.1744 & 181.4289 & 56.2606 \\
\hline & 10 & 99.3846 & 28.0773 & 160.1579 & 48.4134 & 180.9213 & 55.6413 \\
\hline & 20 & 98.8368 & 27.4759 & 159.8028 & 47.9923 & 180.7948 & 55.4886 \\
\hline & 50 & 98.6845 & 27.3121 & 159.7037 & 47.8757 & 180.7594 & 55.446 \\
\hline & 100 & 98.6628 & 27.2889 & 159.6895 & 47.8591 & 180.7544 & 55.4399 \\
\hline & plate & 98.6555 & 27.2811 & 159.6848 & 47.8535 & 180.7527 & 55.4379 \\
\hline
\end{tabular}


The systematic study of the deflection response for shallow spherical and cylindrical panels under a uniform loading condition is finally plotted in Figure $6 \mathrm{a}, \mathrm{b}$, respectively, for different nonlocal parameters and side-to-thickness ratios $a / h$. Based on the histograms of Figure 6, the deflection response seems to be almost unaffected by the $a / h$ ratio, under a fixed nonlocal parameter $\mu$, while being significantly affected by the nonlocal parameter with a gradual increase in flexibility for increasing values of $\mu$ under the same assumption for $a / h$.

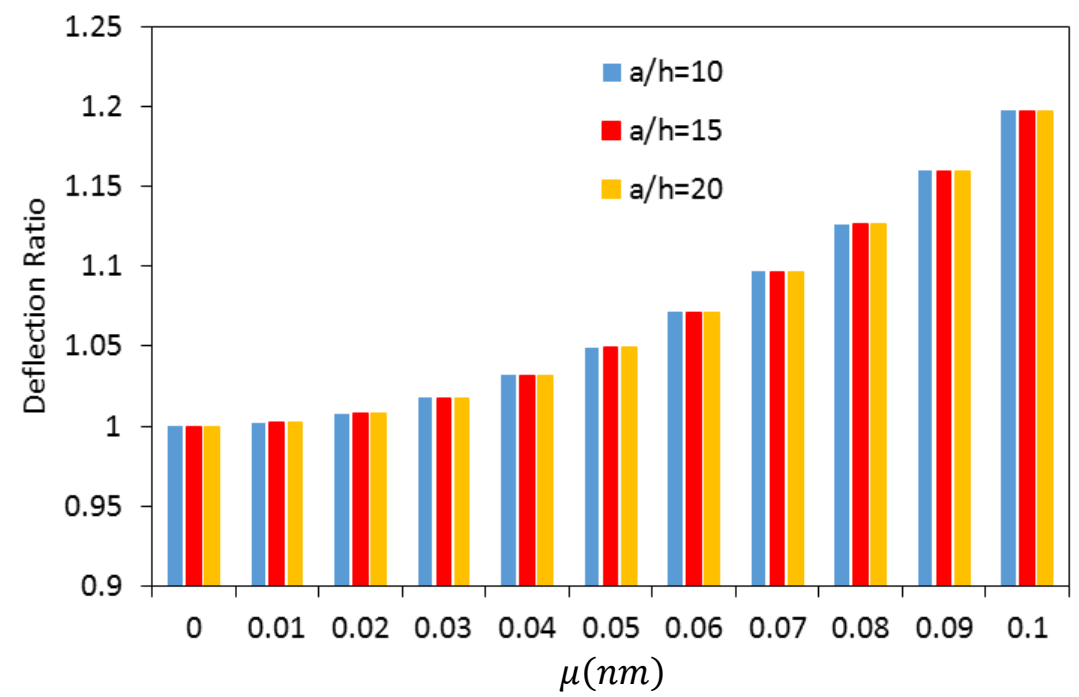

(a)

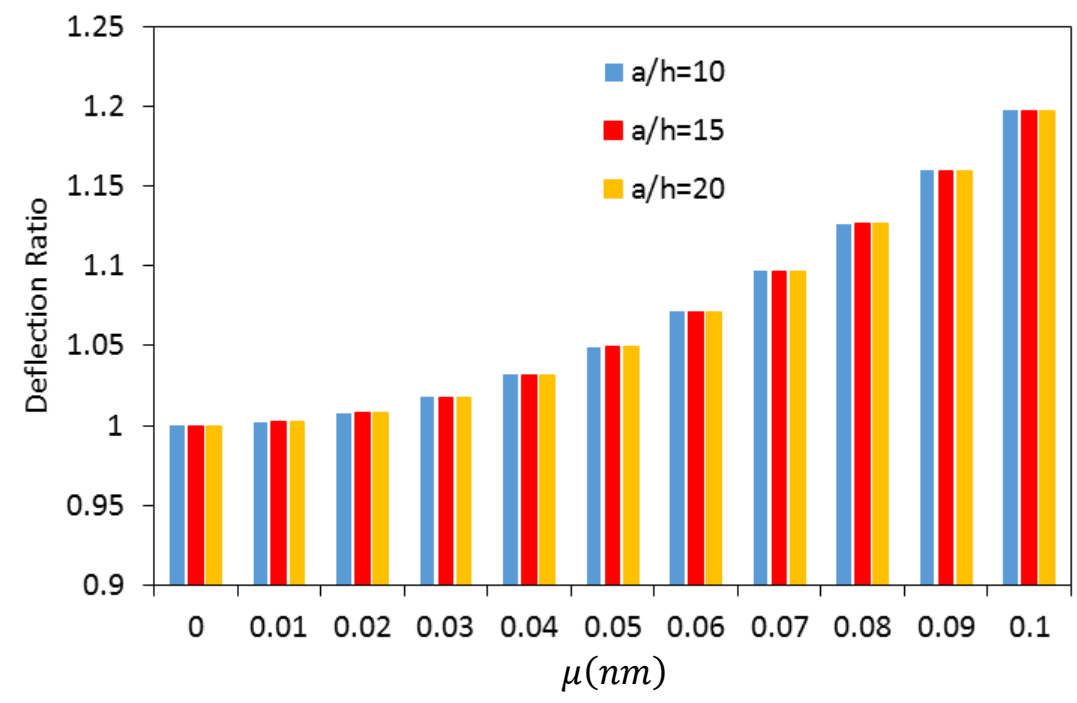

(b)

Figure 6. Effect of the nonlocal parameter and $a / h$ ratio on the deflection ratio of shallow (a) spherical panels with $R_{1}=R_{2}, R / a=20$, and (b) cylindrical panels with $R_{1} / a=20, R_{2} \rightarrow \infty$.

\subsection{Static Analysis of Doubly-Curved Deep Nanoshells}

The same unified formulation is applied in this subsection to study the structural response of doubly-curved deep nanoshells with $R / a \leq 5$. In Figure $7 \mathrm{a}-\mathrm{c}$, we plot the distribution of the non-dimensional stress components throughout the thickness for a deep spherical panel and for a varying nonlocal parameter. According to results in Figure $7 \mathrm{a}-\mathrm{c}$, an increased nonlocal parameter clearly yields a decreased structural stiffness, a decreased natural frequency, and an overall increase in the axial (Figure 7a), longitudinal, and transverse shear (Figure $7 \mathrm{~b}, \mathrm{c}$ ) stress components. More specifically, the transverse shear stress assumes its highest value at the mid-plane, whereby the axial 
and longitudinal shear stresses are reached at the top and bottom sides, while featuring higher values for a shallow spherical panel compared to the deep one. Once again, this variation in stress distribution for different nonlocalities can clearly affect the global stiffness of a nanostructure, and must be carefully accounted for design purposes.

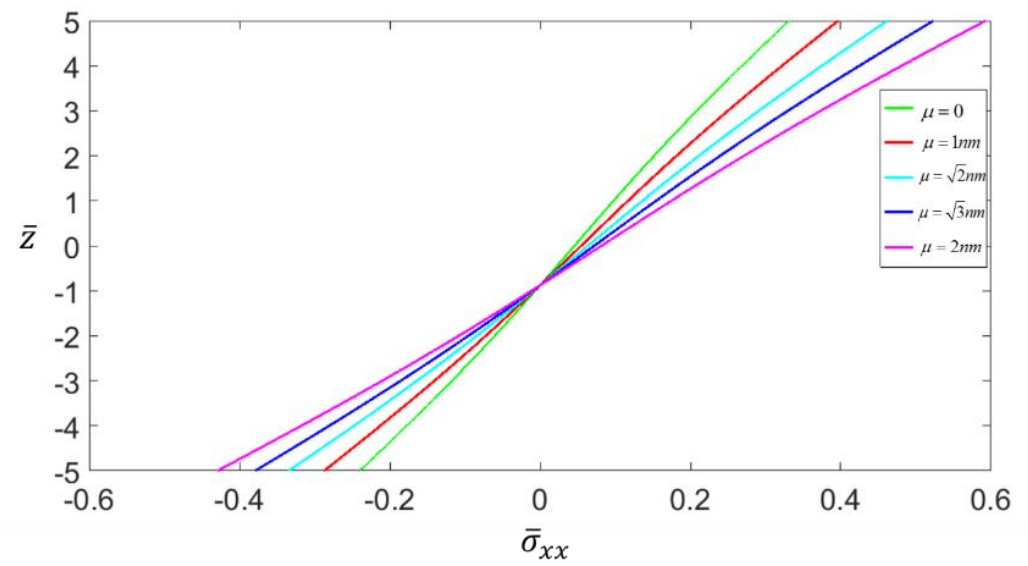

(a)

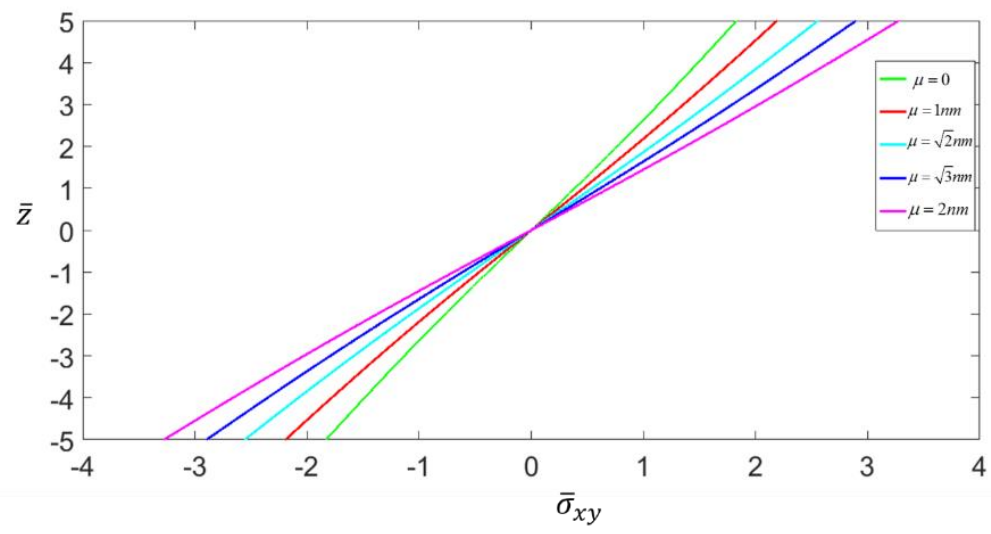

(b)

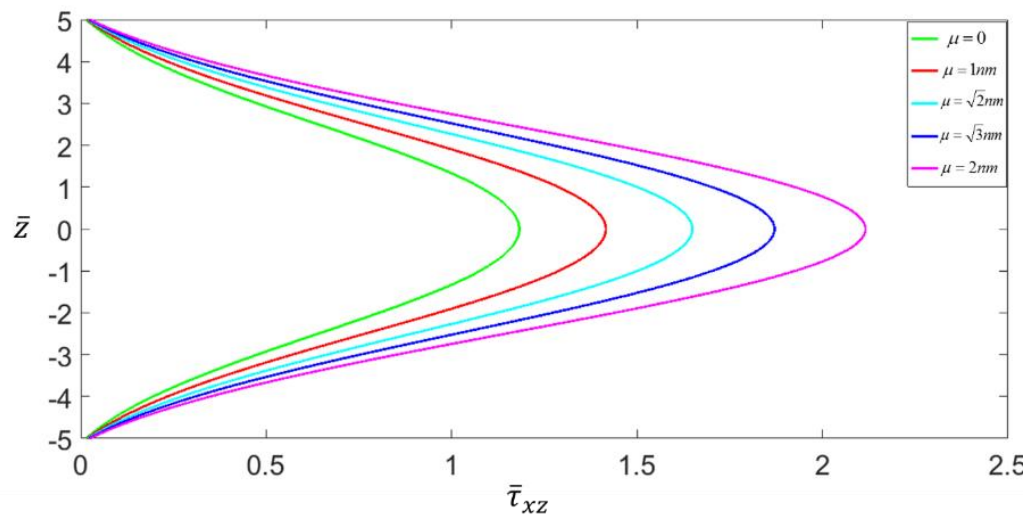

(c)

Figure 7. Variation with the nonlocal parameter of the dimensionless axial stress (a), longitudinal shear stress (b), and transverse shear stress (c), through the thickness of deep spherical panels with $R_{1}=R_{2}, R / a=5, a / h=10$.

Table 5 summarizes the effect of the nonlocal parameter, and length-to-width ratio $b / a$ on the deflection of a thick $(a / h=5)$ and moderately thick $(a / h=10)$ deep spherical panel under a uniform load. Based on the results in Table 5, the non-dimensional deflection clearly increases with the nonlocal 
parameter due to the reduced structural stiffness. In addition, the deformability of the deep spherical panel seems to reduce for an increased $a / h$ ratio and a decreased $b / a$ ratio. Based on a comparative evaluation of Tables 3 and 5, it can be concluded that the deflection of shallow panels is greater than deep panels, or equivalently shallow panels are more flexible than deep counterparts. As plotted in Figure 8, the non-dimensional deflection of the deep spherical panel increases for an increased nonlocal parameter, and length-to-side ratio.

Table 5. Non-dimensional deflection of deep spherical panels $\left(\widetilde{w}=w\left(10^{2} E h^{3}\right) /\left(q_{0} a^{4}\right)\right)$ under a uniform load. $R_{1}=R_{2}, R / a=5$.

\begin{tabular}{ccccccc}
\hline \multirow{2}{*}{$\boldsymbol{\mu}(\boldsymbol{n m})$} & \multicolumn{2}{c}{$\boldsymbol{b} / \boldsymbol{a}=\mathbf{1}$} & \multicolumn{2}{c}{$\boldsymbol{b} / \boldsymbol{a}=\mathbf{2}$} & \multicolumn{2}{c}{$\boldsymbol{b} / \boldsymbol{a}=\mathbf{3}$} \\
\cline { 2 - 7 } & $\boldsymbol{a} / \boldsymbol{h}=\mathbf{5}$ & $\boldsymbol{a} / \boldsymbol{h}=\mathbf{1 0}$ & $\boldsymbol{a} / \boldsymbol{h}=\mathbf{5}$ & $\boldsymbol{a} / \boldsymbol{h}=\mathbf{1 0}$ & $\boldsymbol{a} / \boldsymbol{h}=\mathbf{5}$ & $\boldsymbol{a} / \boldsymbol{h}=\mathbf{1 0}$ \\
\hline 0 & 5.2322 & 4.2624 & 12.0728 & 9.2637 & 14.8348 & 11.0326 \\
0.1 & 6.265 & 5.1038 & 13.5622 & 10.4066 & 16.4617 & 12.2425 \\
0.2 & 9.3633 & 7.6279 & 18.0305 & 13.8352 & 21.3421 & 15.872 \\
0.3 & 14.5273 & 11.8347 & 25.4776 & 19.5495 & 29.4762 & 21.9213 \\
0.4 & 21.7568 & 17.7243 & 35.9036 & 27.5496 & 40.864 & 30.3903 \\
0.5 & 31.0519 & 25.2966 & 49.3085 & 37.8354 & 55.5054 & 41.2791 \\
0.6 & 42.4126 & 34.5516 & 65.6921 & 50.4069 & 73.4004 & 54.5875 \\
0.7 & 55.8388 & 45.4894 & 85.0547 & 65.2642 & 94.5491 & 70.3157 \\
0.8 & 71.3306 & 58.1099 & 107.4 & 82.4072 & 118.95 & 88.4636 \\
0.9 & 88.888 & 72.4131 & 132.72 & 101.8359 & 146.61 & 109.0312 \\
1 & 108.51 & 88.399 & 161.02 & 123.5504 & 177.52 & 132.0185 \\
1.1 & 130.2 & 106.0677 & 192.29 & 147.5506 & 211.68 & 157.4255 \\
1.2 & 153.95 & 125.4192 & 226.55 & 173.8365 & 249.1 & 185.2523 \\
1.3 & 179.77 & 146.4533 & 263.79 & 202.4082 & 289.77 & 215.4988 \\
1.4 & 207.66 & 169.1702 & 304.14 & 233.2656 & 333.69 & 248.165 \\
1.5 & 237.61 & 193.5698 & 347.19 & 266.4088 & 380.87 & 283.2509 \\
1.6 & 269.63 & 219.6522 & 393.37 & 301.8376 & 431.3 & 320.7565 \\
1.7 & 303.71 & 247.4173 & 442.52 & 339.5522 & 484.99 & 360.6818 \\
1.8 & 339.86 & 276.8651 & 494.65 & 379.5526 & 541.93 & 403.0269 \\
1.9 & 378.07 & 307.9957 & 549.76 & 421.8386 & 602.12 & 447.7917 \\
2 & 418.35 & 340.8089 & 607.84 & 466.4104 & 665.56 & 494.9762 \\
\hline
\end{tabular}

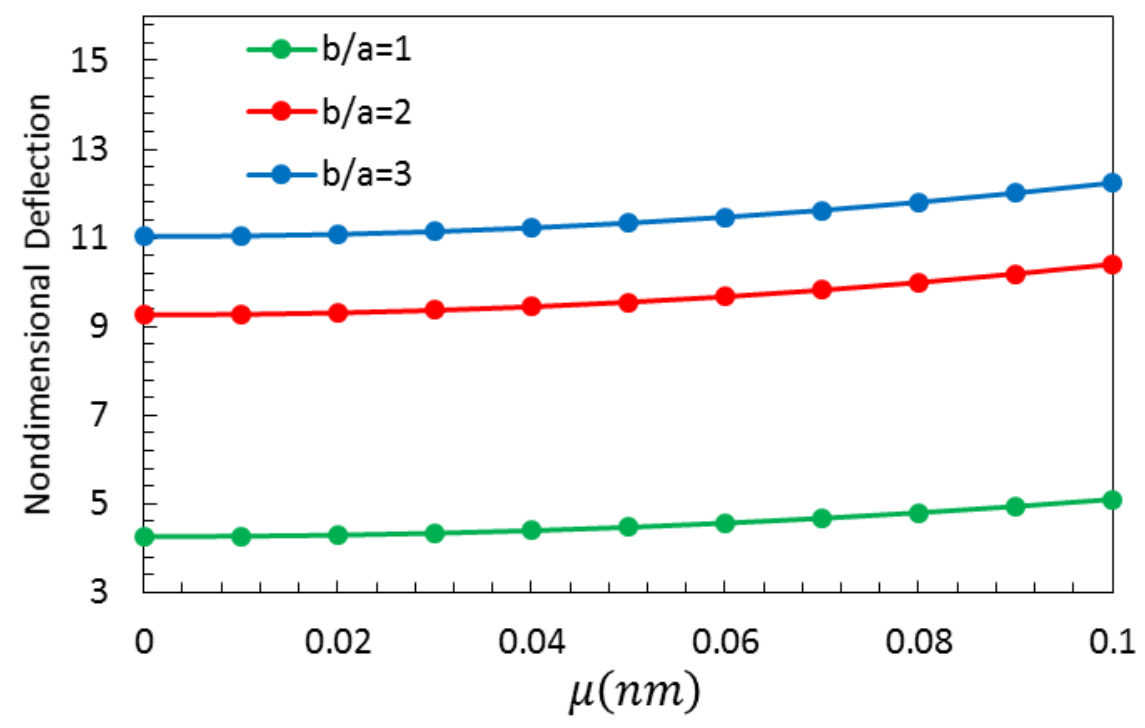

Figure 8. Effect of the nonlocal parameter and $b / a$ ratio on the deflection of deep spherical panels under a uniform load. $R_{1}=R_{2}, R / a=5, a / h=10$. 
A further parametric investigation considers the combined sensitivity of the deflection response to the nonlocal parameter and Poisson's ratio for a thick deep spherical panel with $a / h=5$ under a uniform loading condition. As observed in Figure 9, by increasing the nonlocal parameter, the non-dimensional deflection of the deep spherical panel is increased for each value of Poisson's ratio. Moreover, by increasing the Poisson's ratio, the non-dimensional deflection of the deep spherical panel is gradually decreased.

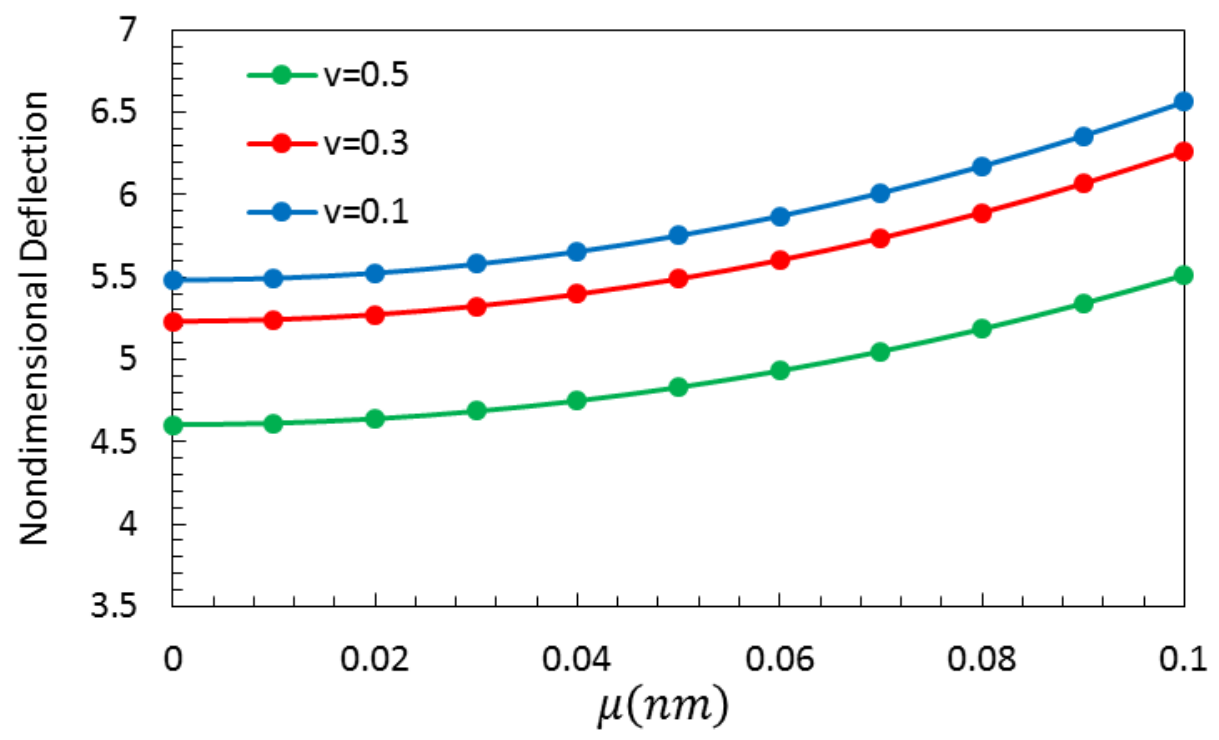

Figure 9. Effect of the nonlocal parameter and Poisson's coefficient on the deflection of deep spherical panels under a uniform load. $R_{1}=R_{2}, R / a=5, a / h=5$.

In the histograms of Figure 10, we quantify the effect of the nonlocal parameter and side-to-thickness ratio, $a / h$, on the deflection ratio of the deep spherical panel under a uniform load. Based on Figure 10, please note that the side-to-thickness ratio does not significantly affect the deflection ratio of the deep spherical panel, independently of the nonlocal parameter, where the only variation in deformability is related to the nonlocal parameter. This justifies the necessity of applying a nonlocal theory instead of the classical elastic ones, which could underestimate the deformability of a nanostructure, in agreement with findings from References [12-14].

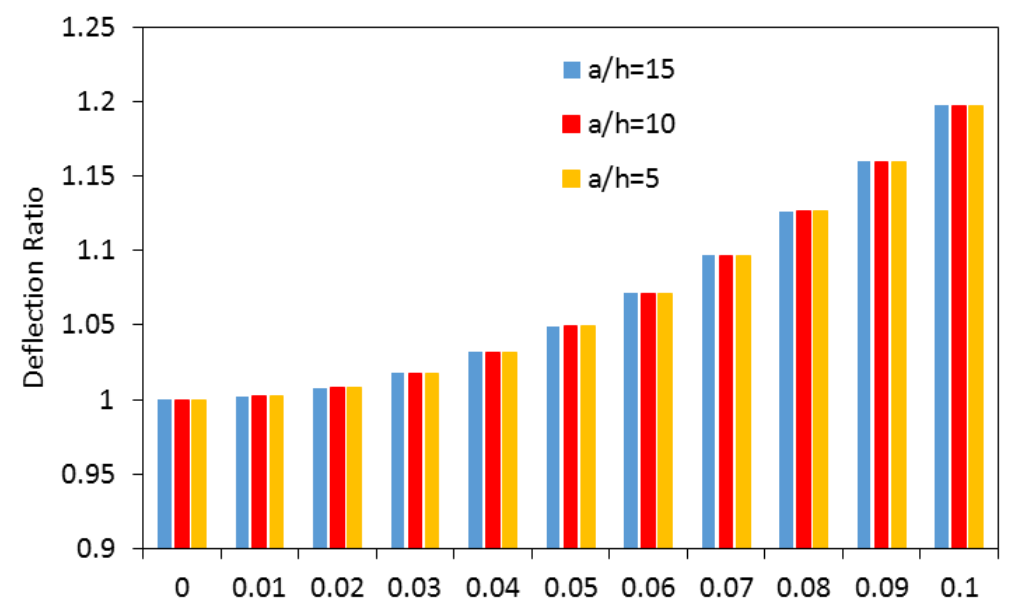

Figure 10. Effect of the nonlocal parameter and $a / h$ ratio on the deflection ratio of deep spherical panels under a uniform load. $R_{1}=R_{2}, R / a=5$. 


\section{Conclusions}

In the present work, we propose a novel nonlocal shear deformation theory to study the bending deflection of isotropic doubly-curved deep nanoshells. The Hamilton's principle is applied to derive the equations of motion, whose solution is determined by means of the Navier method. The proposed formulation is able to handle both thin and thick, shallow and deep nanoshells within a unified framework.

A large parametric investigation is performed systematically to check for the sensitivity of the deflection response for the nonlocal, mechanical, and geometrical parameters, where the following concluding remarks can be summarized.

- An increased nonlocal parameter decreases the stiffness of the isotropic shallow and deep panels, along with a decreased natural frequency, an increased deflection of the nanostructure, and increased stress components.

- An increased value for the side-to-thickness ratio, Poisson's ratio, and length-to-side ratio yields a reduced deflection in the isotropic shallow and deep panel.

- The side-to-thickness ratio does not significantly affect the deflection ratio of shallow and deep panels.

- The axial and longitudinal shear stress components at the top and bottom sides of shallow panels feature higher values than the deep ones.

- Shallow panels are more flexible than deep panels, as visible from their higher deformable response, when compared to deep panels.

Author Contributions: Conceptualization, M.H.D., L.L., R.D. and F.T.; Formal analysis, M.H.D., L.L., R.D. and F.T.; Investigation, M.H.D., L.L. and F.T.; Validation, M.H.D., R.D. and F.T.; Writing-Original Draft, M.H.D., L.L., R.D. and F.T.; Writing-Review \& Editing, R.D. and F.T. All authors have read and agreed to the published version of the manuscript.

Funding: This research received no external funding.

Conflicts of Interest: The authors declare no conflict of interest.

\section{Appendix A}

An explicit definition of parameters in Equation (15) is given below

$$
\begin{aligned}
& \left(A_{4}, A_{5}, A_{6}, A_{7}, A_{8}, A_{9}, A_{10}\right)=\int_{-\frac{h}{2}}^{\frac{h}{2}} Q_{11}\left(1, \xi_{3}, \xi_{3}^{2}, \xi_{3}^{3}, f, f \xi_{3}, f^{2}\right) d \xi_{3} \\
& \left(B_{4}, B_{5}, B_{6}, B_{7}, B_{8}, B_{9}, B_{10}\right)=\int_{-\frac{h}{2}}^{\frac{h}{2}} Q_{12}\left(1, \xi_{3}, \xi_{3}^{2}, \xi_{3}^{3}, f, f \xi_{3}, f^{2}\right) d \xi_{3} \\
& \left(C_{4}, C_{5}, C_{6}, C_{7}, C_{8}, C_{9}, C_{10}\right)=\int_{-\frac{h}{2}}^{\frac{h}{2}} Q_{22}\left(1, \xi_{3}, \xi_{3}^{2}, \xi_{3}^{3}, f, f \xi_{3}, f^{2}\right) d \xi_{3} \\
& \left(D_{4}, D_{5}, D_{6}, D_{7}, D_{8}, D_{9}, D_{10}\right)=\int_{-\frac{h}{2}}^{\frac{h}{2}} Q_{66}\left(1, \xi_{3}, \xi_{3}^{2}, \xi_{3}^{3}, f, f \xi_{3}, f^{2}\right) d \xi_{3} \\
& \left(A_{11}, A_{13}, A_{15}, A_{16}, A_{17}\right)=\int_{-\frac{h}{2}}^{\frac{h}{2}} Q_{11}\left(1+\frac{\xi_{3}}{R_{1}}\right)\left(1,\left(1+\frac{\xi_{3}}{R_{1}}\right),\left(1+\frac{\xi_{3}}{R_{2}}\right), \xi_{3}, f\right) d \xi_{3} \\
& \left(A_{12}, A_{14}, A_{18}, A_{19}\right)=\int_{-\frac{h}{2}}^{\frac{h}{2}} Q_{11}\left(1+\frac{\xi_{3}}{R_{2}}\right)\left(1,\left(1+\frac{\xi_{3}}{R_{2}}\right), \xi_{3}, f\right) d \xi_{3} \\
& \left(B_{11}, B_{13}, B_{15}, B_{16}, B_{17}\right)=\int_{-\frac{h}{2}}^{\frac{h}{2}} Q_{12}\left(1+\frac{\xi_{3}}{R_{1}}\right)\left(1,\left(1+\frac{\xi_{3}}{R_{1}}\right),\left(1+\frac{\xi_{3}}{R_{2}}\right), \xi_{3^{\prime}}, f\right) d \xi_{3} \\
& \left(B_{12}, B_{14}, B_{18}, B_{19}\right)=\int_{-\frac{h}{2}}^{\frac{h}{2}} Q_{12}\left(1+\frac{\xi_{3}}{R_{2}}\right)\left(1,\left(1+\frac{\xi_{3}}{R_{2}}\right), \xi_{3}, f\right) d \xi_{3} \\
& \left(C_{11}, C_{13}, C_{15}, C_{16}, C_{17}\right)=\int_{-\frac{h}{2}}^{\frac{h}{2}} Q_{22}\left(1+\frac{\xi_{3}}{R_{1}}\right)\left(1,\left(1+\frac{\xi_{3}}{R_{1}}\right),\left(1+\frac{\xi_{3}}{R_{2}}\right), \xi_{3}, f\right) d \xi_{3} \\
& \left(C_{12}, C_{14}, C_{18}, C_{19}\right)=\int_{-\frac{h}{2}}^{\frac{h}{2}} Q_{22}\left(1+\frac{\xi_{3}}{R_{2}}\right)\left(1,\left(1+\frac{\xi_{3}}{R_{2}}\right), \xi_{3}, f\right) d \xi_{3}
\end{aligned}
$$




$$
\begin{aligned}
& \left(D_{11}, D_{13}, D_{15}, D_{16}, D_{17}\right)=\int_{-\frac{h}{2}}^{\frac{h}{2}} Q_{66}\left(1+\frac{\xi_{3}}{R_{1}}\right)\left(1,\left(1+\frac{\xi_{3}}{R_{1}}\right),\left(1+\frac{\xi_{3}}{R_{2}}\right), \xi_{3}, f\right) d \xi_{3} \\
& \left(D_{12}, D_{14}, D_{18}, D_{19}\right)=\int_{-\frac{h}{2}}^{\frac{h}{2}} Q_{66}\left(1+\frac{\xi_{3}}{R_{2}}\right)\left(1,\left(1+\frac{\xi_{3}}{R_{2}}\right), \xi_{3}, f\right) d \xi_{3} \\
& \left(G_{11}, G_{12}, G_{13}, G_{14}\right)=\int_{-\frac{h}{2}}^{\frac{h}{2}} Q_{55}\left(1+\frac{\xi_{3}}{R_{1}}\right)^{2}\left(1, f, \frac{d f}{d \xi_{3}},\left(\frac{d f}{d \xi_{3}}\right)^{2}\right) d \xi_{3} \\
& \left(F_{11}, F_{12}, F_{13}, F_{14}\right)=\int_{-\frac{h}{2}}^{\frac{h}{2}} Q_{44}\left(1+\frac{\xi_{3}}{R_{1}}\right)^{2}\left(1, f, \frac{d f}{d \xi_{3}},\left(\frac{d f}{d \xi_{3}}\right)^{2}\right) d \xi_{3}
\end{aligned}
$$

\section{Appendix B}

By substituting Equation (17) into Equation (15), the stiffness matrix is defined as follows

$$
\begin{aligned}
& K(1,1)=-A_{13} \alpha^{2}-D_{13} \beta^{2}-\frac{G_{11}}{R_{1}^{2}} \\
& K(1,2)=-B_{15} \alpha \beta-D_{15} \alpha \beta \\
& K(1,3)=A_{16} \alpha^{3}+B_{16} \alpha \beta^{2}+2 D_{16} \alpha \beta^{2}+\frac{A_{11} \alpha}{R_{1}}+\frac{B_{11} \alpha}{R_{2}} \\
& K(1,4)=-A_{16} y^{*} \alpha^{2}-A_{17} \alpha^{2}-D_{16} y^{*} \beta^{2}-D_{17} \beta^{2}-\frac{G_{13}}{R_{1}} \\
& K(1,5)=-B_{16} y^{*} \alpha \beta-B_{17} \alpha \beta-D_{16} y^{*} \alpha \beta-D_{17} \alpha \beta \\
& K(2,1)=-B_{15} \alpha \beta-D_{13} \alpha \beta \\
& K(2,2)=-C_{14} \beta^{2}-D_{15} \alpha^{2}-\frac{F_{11}}{R_{2}^{2}} \\
& K(2,3)=B_{18} \alpha^{2} \beta+B_{12} \frac{\beta}{R_{1}}+C_{18} \beta^{3}+\frac{C_{12} \beta}{R_{2}}+2 D_{16} \alpha^{2} \beta \\
& K(2,4)=-B_{18} y^{*} \alpha \beta-B_{19} \alpha \beta-D_{17} \alpha \beta-D_{16} y^{*} \alpha \beta \\
& K(2,5)=-C_{18} y^{*} \beta^{2}-C_{19} \beta^{2}-D_{16} y^{*} \alpha^{2}-D_{17} \alpha^{2}-F_{11} \frac{y^{*}}{R_{2}}-\frac{F_{13}}{R_{2}} \\
& K(3,1)=A_{16} \alpha^{3}+B_{16} \alpha \beta^{2}+2 D_{16} \alpha \beta^{2}+\frac{A_{11} \alpha}{R_{1}}+\frac{B_{11} \alpha}{R_{2}} \\
& K(3,2)=B_{18} \alpha^{2} \beta+C_{18} \beta^{3}+2 D_{18} \alpha^{2} \beta+\frac{B_{12} \beta}{R_{1}}+\frac{C_{12} \beta}{R_{2}} \\
& K(3,3)=-A_{6} \alpha^{4}-\frac{A_{5} \alpha^{2}}{R_{1}}-2 B_{6} \alpha^{2} \beta^{2}-\frac{B_{5} \alpha^{2}}{R_{2}}-\frac{B_{5} \beta^{2}}{R_{1}}-C_{6} \beta^{4}-\frac{C_{5} \beta^{2}}{R_{2}}-4 D_{6} \alpha^{2} \beta^{2}-\frac{A_{5} \alpha^{2}}{R_{1}}-\frac{A_{4}}{R_{1}^{2}}-\frac{B_{5} \beta^{2}}{R_{1}}+ \\
& -\frac{B_{4}}{R_{1} R_{2}}-\frac{B_{5} \alpha^{2}}{R_{2}}-\frac{B_{4}}{R_{1} R_{2}}-\frac{C_{5} \beta^{2}}{R_{2}}-\frac{C_{4}}{R_{2}^{2}} \\
& K(3,4)=A_{6} y^{*} \alpha^{3}+A_{9} \alpha^{3}+B_{6} y^{*} \alpha \beta^{2}+B_{9} \alpha \beta^{2}+2 D_{6} y^{*} \alpha \beta^{2}+2 D_{9} \alpha \beta^{2}+A_{5} y^{*} \frac{\alpha}{R_{1}}+A_{8} \frac{\alpha}{R_{1}}+B_{5} y^{*} \frac{\alpha}{R_{2}}+B_{8} \frac{\alpha}{R_{2}} \\
& K(3,5)=B_{6} y^{*} \alpha^{2} \beta+B_{9} \alpha^{2} \beta+C_{6} y^{*} \beta^{3}+C_{9} \beta^{3}+2 D_{6} y^{*} \alpha^{2} \beta+2 D_{9} \alpha^{2} \beta+B_{5} y^{*} \frac{\beta}{R_{1}}+B_{8} \frac{\beta}{R_{1}}+C_{5} y^{*} \frac{\beta^{2}}{R_{2}}+C_{8} \frac{\beta^{2}}{R_{2}} \\
& K(4,1)=-A_{16} y^{*} \alpha^{2}-D_{16} y^{*} \alpha^{2}-A_{17} \alpha^{2}-D_{17} \alpha^{2}-G_{11} \frac{y^{*}}{R_{1}}-\frac{G_{13}}{R_{1}} \\
& K(4,2)=-B_{18} y^{*} \alpha \beta-D_{18} y^{*} \alpha \beta-B_{19} \alpha \beta-D_{19} \alpha \beta \\
& K(4,3)=A_{6} y^{*} \alpha^{3}+A_{5} \frac{y^{*} \alpha}{R_{1}}+B_{6} y^{*} \alpha \beta^{2}+B_{5} \frac{y^{*} \alpha}{R_{2}}+2 D_{6} y^{*} \alpha \beta^{2}+A_{9} \alpha^{3}+A_{8} \frac{\alpha}{R_{1}}+B_{9} \alpha \beta^{2}+B_{8} \frac{\alpha}{R_{2}}+2 D_{9} \alpha \beta^{2} \\
& K(4,4)=-A_{6} y^{* 2} \alpha^{2}-A_{9} y^{*} \alpha^{2}-D_{6} y^{* 2} \beta^{2}-D_{9} y^{*} \beta^{2}-A_{9} y^{*} \alpha^{2}-A_{10} \alpha^{2}-D_{9} y^{*} \beta^{2}-D_{10} \beta^{2}-G_{11} y^{* 2}-G_{13} y^{*} \\
& K(4,5)=-B_{6} y^{* 2} \alpha \beta-B_{9} y^{*} \alpha \beta-D_{6} y^{* 2} \beta \alpha-D_{9} y^{*} \beta \alpha-B_{9} y^{*} \alpha \beta-B_{10} \alpha \beta-D_{9} y^{*} \alpha \beta-D_{10} \alpha \beta \\
& K(5,1)=-B_{16} y^{*} \alpha \beta-D_{16} y^{*} \alpha \beta-B_{17} \alpha \beta-D_{17} \alpha \beta \\
& K(5,2)=-C_{18} y^{*} \beta^{2}-D_{18} y^{*} \alpha^{2}-C_{19} \beta^{2}-D_{19} \alpha^{2}-F_{11} \frac{y^{*}}{R_{2}}-\frac{F_{13}}{R_{2}} \\
& K(5,3)=B_{6} y^{*} \alpha^{2} \beta+B_{5} \frac{y^{*} \beta}{R_{1}}+C_{6} y^{*} \beta^{3}+C_{5} \frac{y^{*} \beta}{R_{2}}+2 D_{6} y^{*} \alpha^{2} \beta+B_{9} \alpha^{2} \beta+B_{8} \frac{\beta}{R_{1}}+C_{9} \beta^{3}+C_{8} \frac{\beta}{R_{2}}+2 D_{9} \alpha^{2} \beta \\
& K(5,4)=-B_{6} y^{* 2} \alpha \beta-B_{9} y^{*} \alpha \beta-D_{6} y^{* 2} \alpha \beta-D_{9} y^{*} \alpha \beta-B_{9} y^{*} \alpha \beta-B_{10} \alpha \beta-D_{9} y^{*} \alpha \beta-D_{10} \alpha \beta \\
& K(5,5)=-C_{6} y^{* 2} \beta^{2}-C_{9} y^{*} \beta^{2}-D_{6} y^{* 2} \alpha^{2}-D_{9} y^{*} \alpha^{2}-C_{9} y^{*} \beta^{2}-C_{10} \beta^{2}-D_{9} y^{*} \alpha^{2}-D_{10} \alpha^{2}-F_{11} y^{*^{2}}-2 F_{13} y^{*}-F_{14}
\end{aligned}
$$

\section{References}

1. Love, A.H. A Treatise on the Mathematical Theory of Elasticity; Cambridge University Press: Cambridge, UK, 1944; Volume 1, p. 952.

2. Sokolnikoff, I.S.; Walker, M. Tensor analysis: Theory and applications. Am. J. Phys. 1952, 20, $249-250$. [CrossRef]

3. Sokolnikoff, I.S. Mathematical Theory of Elasticity; McGraw-Hill: New York, NY, USA, 1956; Volume 83.

4. Timoshenko, S.P.; Woinowsky-Krieger, S. Theory of Plates and Shells; McGraw-Hill: New York, NY, USA, 1959. 
5. Leissa, A.W. Vibration of Shells; NASA: Washington, DC, USA, 1973.

6. Niordson, F. Shell Theory; North-Holland: Amsterdam, The Nederland, 1985.

7. Tornabene, F.; Fantuzzi, N. Mechanics of Laminated Composite Doubly-Curvel Shell Structures: The Generalized Differential Quadrature Method and the Strong Formulation Finite Element Method; Società Editrice Esculapio: Bologna, Italy, 2014.

8. Carrera, E. Multilayered shell theories accounting for layerwise mixed description, Part 1: Governing equations. AIAA J. 1999, 37, 1107-1116. [CrossRef]

9. Carrera, E. Historical review of zig-Zag theories for multilayered plates and shells. Appl. Mech. Rev. 2003, 56, 287-308. [CrossRef]

10. Tornabene, F.; Viola, E.; Inman, D.J. 2-D differential quadrature solution for vibration analysis of functionally graded conical, cylindrical shell and annular plate structures. J. Sound Vib. 2009, 328, 259-290. [CrossRef]

11. Duc, N.D.; Quan, T.Q. Transient responses of functionally graded double curved shallow shells with temperature-Dependent material properties in thermal environment. Eur. J. Mech.-A/Solids 2014, 47, 101-123. [CrossRef]

12. Eringen, A.C. Nonlocal polar elastic continua. Int. J. Eng. Sci. 1972, 10, 1-16. [CrossRef]

13. Eringen, A.C. On differential equations of nonlocal elasticity and solutions of screw dislocation and surface waves. J. Appl. Phys. 1983, 54, 4703-4710. [CrossRef]

14. Eringen, A.C. Nonlocal Continuum Field Theories; Springer Science \& Business Media: New York, NY, USA, 2002.

15. Ramirez, F.; Heyliger, P.R.; Pan, E. Static analysis of functionally graded elastic anisotropic plates using a discrete layer approach. Compos. Part B Eng. 2006, 37, 10-20. [CrossRef]

16. Merdaci, S.; Belghoul, H. High-Order shear theory for static analysis of functionally graded plates with porosities. Comptes Rendus Mécanique 2019, 347, 207-217. [CrossRef]

17. Alibeigloo, A.; Nouri, V. Static analysis of functionally graded cylindrical shell with piezoelectric layers using differential quadrature method. Compos. Struct. 2010, 92, 1775-1785. [CrossRef]

18. Kumar, R.; Mishra, B.; Jain, S. Static and dynamic analysis of smart cylindrical shell. Finite Elem. Anal. Des. 2008, 45, 13-24. [CrossRef]

19. Frikha, A.; Dammak, F. Geometrically non-Linear static analysis of functionally graded material shells with a discrete double directors shell element. Comput. Methods Appl. Mech. Eng. 2017, 315, 1-24. [CrossRef]

20. Mantari, J.L.; Oktem, A.S.; Soares, C.G. Static and dynamic analysis of laminated composite and sandwich plates and shells by using a new higher-Order shear deformation theory. Compos. Struct. 2011, 94, 37-49. [CrossRef]

21. Sahmani, S.; Aghdam, M. Nonlinear instability of hydrostatic pressurized hybrid FGM exponential shear deformable nanoshells based on nonlocal continuum elasticity. Compos. Part B Eng. 2017, 114, 404-417. [CrossRef]

22. Zeighampour, H.; Beni, Y.T.; Karimipour, I. Torsional vibration and static analysis of the cylindrical shell based on strain gradient theory. Arab. J. Sci. Eng. 2016, 41, 1713-1722. [CrossRef]

23. Sahmani, S.; Aghdam, M. Size dependency in axial postbuckling behavior of hybrid FGM exponential shear deformable nanoshells based on the nonlocal elasticity theory. Compos. Struct. 2017, 166, 104-113. [CrossRef]

24. Ke, L.; Wang, Y.; Reddy, J. Thermo-Electro-Mechanical vibration of size-Dependent piezoelectric cylindrical nanoshells under various boundary conditions. Compos. Struct. 2014, 116, 626-636. [CrossRef]

25. Salehipour, H.; Shahsavar, A.; Civalek, O. Free vibration and static deflection analysis of functionally graded and porous micro/nanoshells with clamped and simply supported edges. Compos. Struct. 2019, 221, 110842. [CrossRef]

26. Zeng, S.; Wang, B.; Wang, K. Static stability analysis of nanoscale piezoelectric shells with flexoelectric effect based on couple stress theory. Microsyst. Technol. 2018, 24, 2957-2967. [CrossRef]

27. Arefi, M.; Mohammad-Rezaei Bidgoli, E.; Dimitri, R.; Tornabene, F. Free vibrations of functionally graded polymer composite nanoplates reinforced with graphene nanoplatelets. Aerosp. Sci. Technol. 2018, 81, 108-117. [CrossRef]

28. Jouneghani, F.Z.; Dimitri, R.; Tornabene, F. Structural response of porous FG nanobeams under hygro-Thermo-Mechanical loadings. Compos. Part B Eng. 2018, 152, 71-78. [CrossRef]

29. Arefi, M.; Mohammad-Rezaei Bidgoli, E.; Dimitri, R.; Bacciocchi, M.; Tornabene, F. Nonlocal bending analysis of curved nanobeams reinforced by graphene nanoplatelets. Compos. Part B Eng. 2019, 166, 1-12. [CrossRef] 
30. Arefi, M.; Bidgoli, E.M.-R.; Dimitri, R.; Tornabene, F.; Reddy, J.N. Size-Dependent free vibrations of FG polymer composite curved nanobeams reinforced with graphene nanoplatelets resting on Pasternak foundations. Appl. Sci. 2019, 9, 1580. [CrossRef]

31. Karami, B.; Janghorban, M.; Shahsavari, D.; Dimitri, R.; Tornabene, F. Nonlocal Buckling Analysis of Composite Curved Beams Reinforced with Functionally Graded Carbon Nanotubes. Molecules 2019, $24,2750$. [CrossRef] [PubMed]

32. Malikan, M.; Nguyen, V.B.; Dimitri, R.; Tornabene, F. Dynamic modeling of non-Cylindrical curved viscoelastic single-Walled carbon nanotubes based on the second gradient theory. Mater. Res. Express 2019, 6, 075041. [CrossRef]

33. Arefi, M. Analysis of a doubly curved piezoelectric nano shell: Nonlocal electro-Elastic bending solution. Eur. J. Mech.-A/Solids 2018, 70, 226-237. [CrossRef]

34. Tornabene, F.; Fantuzzi, N.; Viola, E.; Carrera, E. Static analysis of doubly-Curved anisotropic shells and panels using CUF approach, differential geometry and differential quadrature method. Compos. Struct. 2014, 107, 675-697. [CrossRef]

35. Tornabene, F.; Viola, E. Static analysis of functionally graded doubly-Curved shells and panels of revolution. Meccanica 2013, 48, 901-930. [CrossRef]

36. Viola, E.; Tornabene, F.; Fantuzzi, N. Static analysis of completely doubly-Curved laminated shells and panels using general higher-Order shear deformation theories. Compos. Struct. 2013, 101, 59-93. [CrossRef]

37. Liew, K.; Lim, C. A higher-Order theory for vibration of doubly curved shallow shells. J. Appl. Mech. 1996, 63, 587-593. [CrossRef]

38. Amabili, M.; Reddy, J. A new non-Linear higher-Order shear deformation theory for large-Amplitude vibrations of laminated doubly curved shells. Int. J. Non-Linear Mech. 2010, 45, 409-418. [CrossRef]

39. Jouneghani, F.Z.; Dashtaki, P.M.; Dimitri, R.; Bacciocchi, M.; Tornabene, F. First-Order shear deformation theory for orthotropic doubly-Curved shells based on a modified couple stress elasticity. Aerosp. Sci. Technol. 2018, 73, 129-147. [CrossRef]

40. Arefi, M.; Rabczuk, T. A nonlocal higher order shear deformation theory for electro-Elastic analysis of a piezoelectric doubly curved nano shell. Compos. Part B Eng. 2019, 168, 496-510. [CrossRef]

41. Karami, B.; Janghorban, M.; Tounsi, A. Variational approach for wave dispersion in anisotropic doubly-Curved nanoshells based on a new nonlocal strain gradient higher order shell theory. Thin-Walled Struct. 2018, 129, 251-264. [CrossRef]

42. Dindarloo, M.H.; Li, L. Vibration analysis of carbon nanotubes reinforced isotropic doubly-Curved nanoshells using nonlocal elasticity theory based on a new higher order shear deformation theory. Compos. Part B Eng. 2019, 175, 107170. [CrossRef]

43. Karami, B.; Shahsavari, D.; Janghorban, M. On the dynamics of porous doubly-Curved nanoshells. Int. J. Eng. Sci. 2019, 143, 39-55. [CrossRef]

44. Chaudhuri, R.A.; Kabir, H.R. On analytical solutions to boundary-Value problems of doubly-Curved moderately-Thick orthotropic shells. Int. J. Eng. Sci. 1989, 27, 1325-1336. [CrossRef]

45. Tornabene, F.; Fantuzzi, N.; Bacciocchi, M. On the mechanics of laminated doubly-Curved shells subjected to point and line loads. Int. J. Eng. Sci. 2016, 109, 115-164. [CrossRef]

46. Ghayesh, M.H.; Farokhi, H. Nonlinear mechanics of doubly curved shallow microshells. Int. J. Eng. Sci. 2017, 119, 288-304. [CrossRef]

47. Zaoui, F.Z.; Ouinas, D.; Tounsi, A. New 2D and quasi-3D shear deformation theories for free vibration of functionally graded plates on elastic foundations. Compos. Part B Eng. 2019, 159, 231-247. [CrossRef]

48. Mantari, J.; Oktem, A.; Soares, C.G. A new higher order shear deformation theory for sandwich and composite laminated plates. Compos. Part B Eng. 2012, 43, 1489-1499. [CrossRef]

49. Reddy, J.N. Mechanics of Laminated Composite Plates and Shells: Theory and Analysis; CRC Press: Boca Raton, FL, USA; London, UK; New York, NY, USA; Washington, DC, USA, 2003.

50. Romano, G.; Barretta, R.; Diaco, M. On nonlocal integral models for elastic nano-Beams. Int. J. Mech. Sci. 2017, 131-132, 490-499. [CrossRef]

51. Barretta, R.; Ali Faghidian, S.; Luciano, R.; Medaglia, C.M.; Penna, R. Free vibrations of FG elastic Timoshenko nano-Beams by strain gradient and stress-Driven nonlocal models. Compos. Part B Eng. 2018, 154, $20-32$. [CrossRef] 
52. Romano, G.; Luciano, R.; Barretta, R.; Diaco, M. Nonlocal integral elasticity in nanostructures, mixtures, boundary effects and limit behaviours. Contin. Mechancis Thermodyn. 2018, 30, 641-655. [CrossRef]

53. Pinnola, F.P.; Ali Faghidian, S.; Barretta, R.; Marotti de Sciarra, F. Variationally consistent dynamics of nonlocal gradient elastic beams. Int. J. Eng. Sci. 2020, 149, 103220. [CrossRef]

54. Reddy, J.N. A simple higher-Order theory for laminated composite plates. J. Appl. Mech. 1984, 51, 745-752. [CrossRef]

55. Reddy, J.; Liu, C. A higher-Order shear deformation theory of laminated elastic shells. Int. J. Eng. Sci. 1985, 23, 319-330. [CrossRef]

56. Reddy, J.N. Energy and Variational Methods in Applied Mechanics: With an Introduction to the Finite Element Method; Wiley: New York, NY, USA, 1984.

57. Reddy, J.N. An Introduction to the Finite Element Method; McGraw-Hill Book Company: New York, NY, USA, 1984.

58. Ferreira, A.; Roque, C.; Martins, P. Analysis of composite plates using higher-Order shear deformation theory and a finite point formulation based on the multiquadric radial basis function method. Compos. Part B Eng. 2003, 34, 627-636. [CrossRef]

59. Ferreira, A.; Roque, C.; Jorge, R. Analysis of composite plates by trigonometric shear deformation theory and multiquadrics. Comput. Struct. 2005, 83, 2225-2237. [CrossRef]

60. Xiang, S.; Wang, K.-M.; Sha, Y.-D.; Shi, H. Analysis of isotropic, sandwich and laminated plates by a meshless method and various shear deformation theories. Compos. Struct. 2009, 91, 31-37. [CrossRef]

61. Bhimaraddi, A. Three-Dimensional elasticity solution for static response of orthotropic doubly curved shallow shells on rectangular planform. Compos. Struct. 1993, 24, 67-77. [CrossRef]

(C) 2020 by the authors. Licensee MDPI, Basel, Switzerland. This article is an open access article distributed under the terms and conditions of the Creative Commons Attribution (CC BY) license (http://creativecommons.org/licenses/by/4.0/). 